\title{
Seeing the future: predictive control in neural models of ocular
} accommodation

4 Jenny C. A. Read ${ }^{1}$, Christos Kaspiris-Rousellis ${ }^{1}$, Toby S. Wood ${ }^{2}$, Bing Wu ${ }^{3}$, Björn N.S.

$5 \quad$ Vlaskamp ${ }^{3}$, Clifton M. Schor ${ }^{4}$

6 1. Biosciences Institute, Newcastle University, NE2 4HH

7 2. School of Mathematics, Statistics \& Physics, Newcastle University, NE1 7RU

8 3. Quantified Experience, Magic Leap Inc

9 4. School of Optometry, University of California at Berkeley

12 Ocular accommodation is the process of adjusting the eye's crystalline lens so as to bring the retinal image into sharp focus. The major stimulus to accommodation is therefore retinal defocus, and in essence, the job of accommodative control is to send a signal to the ciliary muscle which will minimise the magnitude of defocus. In this paper, we first provide a tutorial introduction to control theory to aid vision scientists without this background. We then present a unified model of accommodative control that explains properties of the accommodative response for a wide range of accommodative stimuli. Following previous work, we conclude that most aspects of accommodation are well explained by dual integral control, with a "fast" or "phasic" integrator enabling response to rapid changes in demand, which hands over control to a "slow" or "tonic" integrator which maintains the response to steady demand. Control is complicated by the sensorimotor latencies within the system, which delay both information about defocus and the accommodation changes made in response, and by the sluggish response of the motor plant. These can be overcome by incorporating a Smith predictor, whereby the system predicts the delayed sensory consequences of its own motor actions. For the first time, we show that critically-damped dual integral control with a Smith predictor accounts for adaptation effects as well as for the gain and phase for sinusoidal oscillations in demand. In addition, we propose a novel proportional-control signal to account for the power spectrum of accommodative microfluctuations during steady fixation, which may be important in hunting for optimal focus, and for the nonlinear resonance observed for low-amplitude, high-frequency input. Complete Matlab/Simulink code implementing the model is provided at https://doi.org/10.25405/data.ncl.14945550. 
Accommodation refers to the ability of the eye to change its focus between near and far distances, so as to ensure that images remain in sharp focus at the fovea across a wide range of object distances. This is achieved by changes in the convexity of the intra-ocular lens, brought about by contraction of the ciliary muscle (Figure 1). To focus on distant objects, the ciliary muscle is relaxed, the lens curvature and thus its optical power is minimal; to focus on near objects, the ciliary muscle contracts, the lens curvature increases and so does its optical power.

44

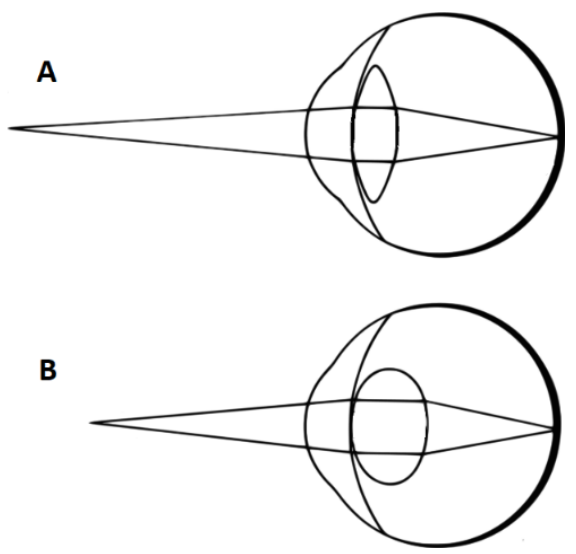

Figure 1. (A) Accommodating on a distant object. When the ciliary muscle is slack, tension in the suspensory zonules is released and the intra-ocular crystalline lens flattens, enabling distant objects to appear in focus on the retina (for an emmetropic eye). Light from a nearby object, such as shown, is therefore out of focus. (B) Accommodating on a nearby object. The ciliary muscle has contracted, increasing the curvature of the lens (blue arrows) in order to bring nearby objects into focus. Not to scale. Image: Pearson Scott Foresman, public domain.

A full understanding of this process requires a knowledge of (i) the optical and biomechanical properties of the eye; (ii) how the required accommodative response is derived from retinal and extra-retinal cues; and (iii) the neural signals controlling the ciliary muscle to bring about this response. In this paper, we concentrate on the third of these.

In Section 1, we discuss the basic structure of models of neural control of accommodation. A key goal of this section is to provide a clear review of the subject, introducing concepts and summarizing previous work in a way which is accessible to vision scientists without a 
background in classical control theory. Accordingly, this section acts as a tutorial to bring such readers up to speed.

The core of accommodative control is a negative feedback loop attempting to null the error between accommodative demand, i.e. the accommodation at which the fixated object will be in sharp focus, and response, i.e. the accommodation actually adopted. We introduce the Smith predictor (Miall et al., 1993; Smith, 1957), which incorporates a forward model of the motor plant to predict the eye's response to a motor command (and which might, in principle, also try to extrapolate the future sensory demand, though our model will not). Predictive models stand in contrast to models which do not take account of the sensory consequences of the body's own motor actions, and which are thus vulnerable to instabilities caused by the finite latencies within the control system.

Armed with this background, in Section 2 we discuss the evidence that accommodation uses a Smith predictor, and examine empirical constraints on the model parameters. We aim to produce a model which can account for behavior in both steady-state and smooth tracking, including accommodative lag/lead, adaptation, critical damping, and Bode plots of gain and phase. (Extending the model to reproduce dynamics of the step response (Bharadwaj \& Schor, 2005, 2006; Schor \& Bharadwaj, 2006, 2004) will be covered in a subsequent paper.)

This analysis leads us to conclude that accommodative control most likely incorporates a predictor, in order to avoid instabilities due to the sensorimotor latency. Again, by "predictor", we mean a forward model to predict the effect of commanded accommodation changes on the visual input, not necessarily anything that predicts changes in accommodative demand.

An oft-neglected component of accommodation models is the effect of noise in both closedloop and open-loop mode. Noise is manifest as small ( $\sim 0.25 \mathrm{D})$ microfluctuations in the steadystate response (Campbell et al., 1959a; Kotulak \& Schor, 1986b). We include a noise source, modelled as Gaussian noise added to the defocus signal. The presence of noise adds a further important constraint on model design. Predictive models have an internal feedback loop via a virtual plant as well as an external feedback loop via the ocular plant. Thus they can end up amplifying internal noise when run in open-loop mode. This is not observed empirically; in 
than open-loop mode. Avoiding open-loop resonances places further constraints on model parameters.

We conclude that all constraints can be simultaneously satisfied, and that accommodation can be modelled successfully as a predictive system with integral control, but that there are fairly tight constraints on the gain and time-constant of the integral controller in order for the system to be consistent with empirical data for step and smooth tracking. Following previous work, we add a slow, second-order integral controller to account for adaptation effects, and show that care is required when using this dual-control with predictive models.

102 Our inclusion of noise leads us to propose adding one further component to the model 103 developed so far: namely a non-predictive proportional-control signal, clipped at low values.

104 We originally rejected non-predictive control because it is prone to closed-loop resonances at 105 particular frequencies. This is because the phase of the cycle where demand is high causes an increase in accommodation designed to null the defocus error, but - due to the latency - by the time the increase in accommodation has taken effect, the demand cycle has moved on to a phase where demand is low, and so the increase in accommodation in fact enhances the defocus error, causing a larger change in accommodation in the next cycle, and so on. We can limit the destabilizing effect of this signal by making it saturate at low values. This ensures that it has

111 little influence on accommodation in general, which remains dominated by the predictive 112 integral control discussed above. However, the closed-loop resonance associated with non113 predictive control remains detectable for small changes in demand. This amplifies noise within

114 particular bandwidths, and means that the microfluctuations in the steady-state response show 115 a peak at frequencies just over $1 \mathrm{~Hz}$, as observed. Opening the loop cuts the feedback pathway 116 generating the resonance, explaining why this peak in the microfluctuation power spectrum is 117 much less prominent in open-loop mode. The saturating proportional signal also accounts for 118 the non-linear resonance observed when accommodation tracks low-amplitude - but not highamplitude - sinusoidal oscillations in demand.

121 Putting these different components together results in a model where accommodation is 122 controlled by the sum of four separate neural signals. The model has a total of ten parameters 123 (Table 2), most of which are quite tightly constrained by the data. In Section 3, we present 
simulation results demonstrating that this model can account simultaneously for a wide range of observations.

\section{Methods}

"A complex system that works is invariably found to have evolved from a simple system that worked. A complex system designed from scratch never works and cannot be patched up to make it work. You have to start over with a working simple system." - Gall's Law (Gall, 1977).

In the spirit of Gall's Law, we begin with the simplest possible conceptual model of accommodation (Figure 2). Viewed as a whole, the model has one input, accommodative demand, corresponding to the vergence of light rays from the object we wish to look at. This is measured in diopters; the demand in diopters corresponds to the reciprocal of the distance in metres from the eye. For an infinitely far object, the demand is $0 \mathrm{D}$; for an object at $50 \mathrm{~cm}$, the demand is $2 \mathrm{D}$.

143 The model also has one output, ocular accommodation. When the eye is correctly 144 accommodated, the accommodation will be equal to the demand so that the image is in focus 145 on the posterior receptor layer of the retina. Defocus is the difference between the accommodative demand and the ocular accommodation, all measured in diopters. It acts as an error signal to the model. As discussed in the Introduction, we assume that defocus is a single, signed value which is somehow computed by the visual system from the retinal image (e.g. using blur, higher-order aberrations, longitudinal chromatic aberration etc (Burge \& Geisler,

150 2011; Cholewiak et al., 2018; Fincham, 1951; Kruger et al., 1993; Seidemann \& Schaeffel, 2002; Wilson et al., 2002)) and represented as a neural error signal; how this is achieved is

152 beyond the scope of this paper. In our sign convention, positive defocus error means that the eye is not accommodating enough, i.e. the eye is focusing on a point more distant than the object of interest, so the ocular image is focused behind the retina. Positive defocus error should therefore stimulate an increase of accommodation. The accommodative control system takes 
signal is then fed into the ocular plant, meaning the ciliary muscle, lens and other components. Changes in the neural signal thus change the ocular accommodation, which in turn affects the defocus error (since defocus is demand minus accommodation). The accommodative control system is designed to adjust accommodation so as to minimise the defocus error signal (Toates, 1972). Thus, this is a negative feedback system.

162

163 In any negative feedback system, one faces the question of how to choose the control signal so as to minimize the error. One obvious form of error correction is to make the corrective signal proportional to the error. For example, a very simple form of automotive cruise control might make acceleration proportional to the difference between the current and the desired speed. Other widely-used possibilities are to integrate the error over time, or to anticipate changes by including a term scaling with the derivative. Together, control systems of this type are called PID (proportional-integral-derivative) controllers.

In reality, of course, defocus is not the only cue to accommodation (Heath, 1956b; Maddox, 1893). One additional component that we discuss below and include in our models is the system's bias towards a particular baseline or resting accommodation (see (Rosenfield et al., 1993a) for a review). Factors which for simplicity we neglect in this paper include pictorial cues to distance, sensed proximity and crosslinks from the vergence system. However, defocus is the only cue which is itself altered by accommodation, and thus the cue intrinsic to the negative feedback loop.

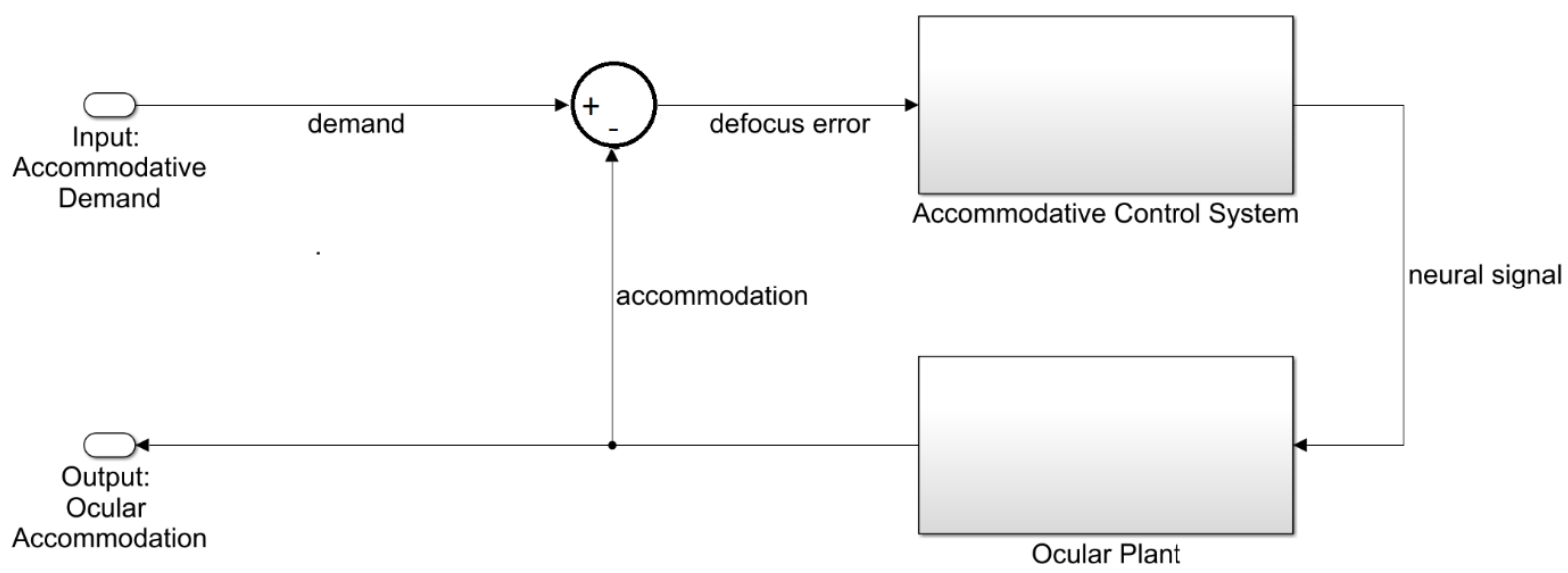


Figure 2. Conceptual model of accommodation. All accommodative models in the literature fit into this basic schematic. There is a feedback loop, whereby the output (accommodation) affects the input to the control system. The blocks labelled Accommodative Control System and Ocular Plant are shown here as "black boxes" which take inputs and yield outputs, without showing how the output is computed. The input to the overall system is the accommodative demand, reflecting the distance of the fixated object, and the output is the ocular accommodation, i.e. where the eye is focused. Defocus error is the

Modelling neural signals as if they were in diopters

190 In this initial part of the paper we will keep the discussion as general as possible, without committing to a particular model of the Ocular Plant or Accommodative Control System blocks shown in Figure 2. However, one detail is worth noting. Without loss of generality we will set the overall gain of the plant to 1 , meaning that it passes a constant signal unchanged. In reality, the neural signal is encoded in spikes per second, and the output of the ocular plant is accommodation in diopters. There must therefore be a gain or conversion factor within the neural signal which converts spikes per second into diopters, taking into account the biomechanical gain of the plant (Gamlin et al., 1994). Without loss of generality, we can fold this conversion factor into our neural signals. Thus by setting the plant gain to 1 , we represent all the neural signals in the model as if they were diopters. This makes them particularly simple to interpret.

203 The model shown in Figure 2 is "closed-loop": that is, the input to the accommodative control system (defocus error) is affected by its output (ocular accommodation). As discussed, this forms a negative feedback loop, in which increases in defocus error stimulate changes in accommodation that in turn reduce defocus error.

208 Figure 3 shows the equivalent open-loop system, in which the output of the system has no effect on its defocus error. It might seem impossible to "cut the wire" in this way in the living eye, but in fact all that is required to examine the open-loop mode is to make the optical error

211 signal independent of the accommodative response. There are two main ways in which this can

212 be done. First, by measuring accommodation and optically adding the current accommodation

213 state onto the current input demand, as shown by the red text in Figure 4. The eye's own optics

214 then effectively removes accommodation, so that the error signal forming the input to the visual 215 system is simply the demand applied by the experimenter, independent of the accommodative 
216 response. A positive non-zero open-loop error signal continues to stimulate increases of the

217 accommodation response until it reaches saturation, reminiscent of a dog chasing its tail.

219 Alternatively, the optical error signal can be set to zero by using a pinhole pupil. Through small

220 pinholes, objects appear slightly blurred due to diffraction, but critically this blur is virtually

221 independent of the stimulus accommodative demand or the ocular accommodation. Pinholes

222 do not cause a "dog chasing tail" accommodative response, but rather accommodation tends to

223 assume its resting state. This suggests that the visual system experiences images seen through

224 pinholes as having zero defocus. Thus, viewing through pinholes is a special case of open-loop

225 in which the input is effectively clamped to zero regardless of output.

226
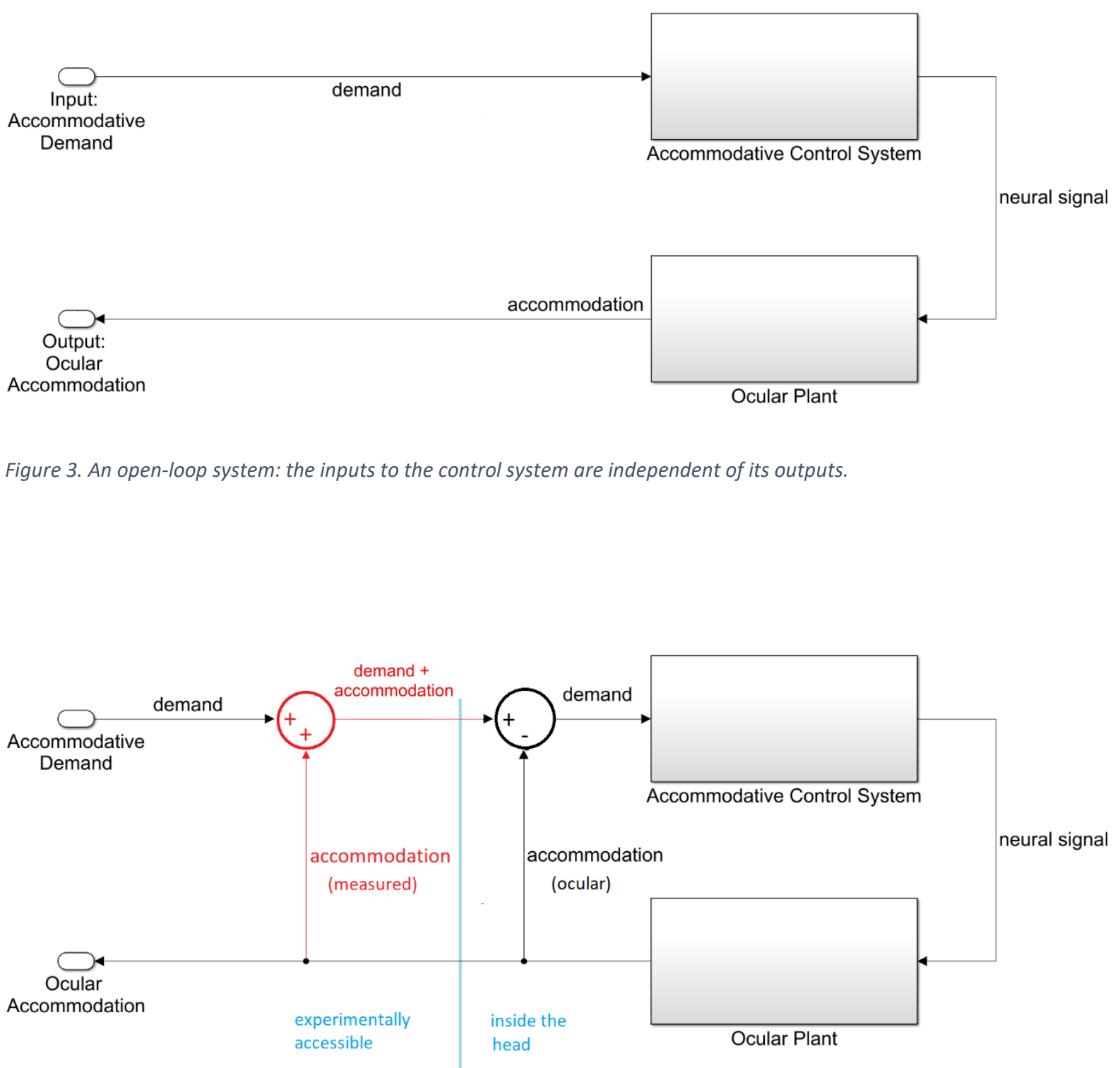
Figure 4. Accommodation driven in open-loop mode by adding the measured accommodation onto the input demand (red).

The signal labelled "accommodation (ocular)" is the actual accommodation state of the eye, which is, as always, subtracted optically in the eye to yield defocus. The signal labelled "accommodation (measured)" is the value measured by the experimenter, which is artificially added onto the original demand signal at the red summation block. Ideally, the measured accommodation should be identical to the actual accommodation, and so the ocular accommodation is perfectly cancelled out by the addition of the measured accommodation. Thus, the error signal input to the block labelled Accommodative Control System remains equal to the applied demand, regardless of the accommodative response. This system is therefore equivalent to the simpler one shown in Figure 3 and is so operated in open-loop mode. Effectively, the red measured accommodation signal "cuts the loop" represented by the ocular accommodation. In this figure, the blue line separates signals which are outside the head and thus accessible for non-invasive experimental manipulation (the original demand and the measured accommodation signal which is added onto it) from signals which are in the eye/brain and thus not easily manipulable.

246 At this point, we note that vision scientists may not be familiar with the classical control systems approach taken in this paper. This section aims to provide a bare-bones introduction to enable such readers to follow subsequent sections. Table 1 provides a reference for all the symbols used throughout the paper.

252 Linear systems are those whose outputs for a linear combination of inputs are the same as a 253 linear combination of individual responses to those inputs. For example, in Figure 2, if the system were linear, then if demand timecourse $d_{1}(\mathrm{t})$ elicited accommodation response $a_{1}(\mathrm{t})$, and demand $d_{2}(\mathrm{t})$ elicited $a_{2}(\mathrm{t})$, the response to a new demand made up of a weighted sum of these two timecourses, $w_{1} d_{1}(\mathrm{t})+w_{2} d_{2}(\mathrm{t})$ would be $w_{1} a_{1}(\mathrm{t})+w_{2} a_{2}(\mathrm{t})$. A time-invariant system is one where the same input, delayed by a time $T$, will always elicit the same response, also delayed by a time $T$. Thus if demand $d_{l}(t)$ elicited accommodation response $a_{1}(t)$, demand $d_{1}(t$ $T)$ would elicit accommodation response $a_{1}(t-T)$.

261 Where a system is both linear and time-invariant (LTI), its response can be analysed using 262 Laplace transforms of the variables. The Laplace transform turns integral and differential equations into polynomial equations which are much easier to solve. Time-domain functions are converted into Laplace-domain functions of a complex frequency variable $s$. We assume that all signals are zero for times before $\mathrm{t}=0$, and write the Laplace transform of a signal $f(t)$ as $F(s)$, where 


$$
F(s)=\int_{0}^{\infty} d t f(t) e^{-s t}
$$

We will adopt the convention where when a lower-case variable represents a function of time $t$, the corresponding upper-case denotes its Laplace transform as a function of $s$. The Laplace transform is closely related to the Fourier transform with which vision scientists are typically more familiar, with $s$ representing a complex version of angular temporal frequency: $s=j \omega$ (where we use $j$ for the square root of -1 throughout).

In a circuit diagram like Figure 2, the effect of an LTI block is simply to reweight the amplitude, and/or shift the phase, of each frequency in the input. This means that each LTI block can be written simply in terms of its complex transfer function $H(s)$. As discussed in more detail below, a transfer function $H(s)$ is a kind of gain, since it is the ratio of the output to the input, for each frequency $s$. For example, consider a transport delay block, whose effect is to delay the input signal by a latency $T$, and which thus shifts the phase of each frequency. If the input signal is $i(t)$, the output after delay is $o(t)=i(t-T)$. Substituting this into Equation 1, we find that

$$
O(s)=\int_{0}^{\infty} d t o(t) e^{-s t}=\int_{0}^{\infty} d t i(t-T) e^{-s t}=\int_{-T}^{\infty} d t i(t) e^{-s t-s T}=e^{-s T} I(s)
$$

where we used the fact that $i(t)=0$ for $t<0$. Thus, the transfer function of a transport delay block is $H(s)=\exp (-s T)$.

In LTI systems, one can do algebra on the Laplace transforms in the usual way. The transfer function for several LTI systems in parallel is the sum of the individual transfer functions, while the transfer function for several LTI systems in series is the product of the transfer functions for the individual systems.

295 When viewing through pinholes, although the demand is zero, accommodation tends not to be zero but to converge on a "rest focus", $a_{\mathrm{RF}}$, generally of around 1.4D (Leibowitz \& Owens, 1978; Rosenfield et al., 1993b). A similar default focus is also observed in darkness. To account 
for this, we assume that the accommodative control system adds onto the signal computed from defocus a constant "bias" signal. Because we have normalized neural signals to be expressed in diopters, setting this bias signal equal to the rest focus ensures that accommodation returns to the rest focus if the defocus error is clamped at zero.

This bias signal leads to a small complication, because it technically violates the assumption that all signals are zero for $t<0$. To handle this, we express both accommodation and demand relative to the rest focus. We define $A(s)$ to be the Laplace transform, not of accommodation itself, but of accommodation relative to rest focus, $a(t)-a_{R F}$. Similarly $D(s)$ is the Laplace transform of demand relative to rest focus, $d(t)-a_{R F}$. With this trick, we can then analyse the system in the Laplace domain as if there were no bias signal $\left(a_{\mathrm{RF}}=0\right)$, and at the end simply add $a_{\mathrm{RF}}$ back on to demand and accommodation when we move back to the time domain. All the analyses in this paper use this approach.

\section{Open- and closed-loop transfer functions}

In Figure 3, where accommodation is driven in open-loop mode, we have

$$
A(s)=P(s) B(s) D(s)
$$

where $B(s)$ is the transfer function representing the brain's accommodative control system and $P(s)$ that representing the ocular plant. As described in the previous section, $A(\mathrm{~s})$ and $D(\mathrm{~s})$ are the Laplace transforms of accommodation and demand relative to rest focus. The open-loop transfer function relating output $A(\mathrm{~s})$ (accommodation) to input $D(\mathrm{~s})$ (demand) is thus

$$
H_{\text {open }}(s)=\frac{A(s)}{D(s)}=P(s) B(s)
$$

In the closed-loop mode shown in Figure 2, the input to the accommodative control system is defocus error, $\mathrm{E}(\mathrm{s})=\mathrm{D}(\mathrm{s})-\mathrm{A}(\mathrm{s})$. We therefore now have

$$
A(s)=H_{\text {open }}(s)[D(s)-A(s)]
$$

and thus derive the closed-loop transfer function:

$$
H_{\text {closed }}(s)=\frac{H_{\text {open }}(s)}{1+H_{\text {open }}(s)}
$$


where

$$
A(s)=H_{\text {closed }}(s) D(s)
$$

331 This relationship between the open- and closed-loop transfer functions is a standard result for 332 a feedback loop like the on e in Figure 2.

335 LTI theory shows that the steady-state response is obtained by evaluating the system at $s=0$ (zero frequency). So, if we apply a constant demand $d_{\text {ss }}$ in closed-loop mode, we have

$$
A(0)=H_{\text {closed }}(0) D(0)
$$

where $D(0)=d_{\mathrm{ss}}-a_{\mathrm{RF}}$ and $\mathrm{A}(0)=a_{\mathrm{ss}}-a_{\mathrm{RF}}$ (recalling that accommodation and demand are defined relative to rest focus $\left.a_{\mathrm{RF}}\right)$. From Equation 4, we can write $H_{\text {closed }}(0)$ in terms of $H_{\text {open }}(0)$. It will be convenient to introduce the notation $G_{\text {open }}$ for $H_{\text {open }}(0)$, i.e. the open-loop steady-state gain of the system. Putting this together with Equation 4 and Equation 5, we find that accommodation will eventually be

$$
a_{S S}=a_{R F}+\frac{G_{\text {open }}}{1+G_{\text {open }}}\left(d_{S S}-a_{R F}\right)
$$

The steady-state defocus error is

$$
d_{s S}-a_{s S}=\frac{d_{s S}-a_{R F}}{1+G_{\text {open }}}
$$

Equation 7 shows that - regardless of the control system or plant - the defocus error will be zero when the demand is equal to the rest focus. This is natural enough, since the rest focus is the value to which the system is biased.

353 However, for other demands, the steady-state error is not zero. When the demand is nearer than

354 the rest focus, the accommodative response remains further than the demand, a situation referred to as accommodative lag. Conversely when demand is further than rest, accommodation is nearer than demand; this is accommodative lead.

358 Importantly, the amount of the error depends on the steady-state open-loop gain $\mathrm{G}_{\mathrm{open}}$. This demonstrates an important property of negative-feedback systems which attempt to minimise 
error: small error requires high open-loop gain. Since we have set the gain of the plant to 1

361 (without loss of generality, as noted above), the gain $G_{\text {open }}$ is set entirely by the brain's accommodative control system. Empirically, accommodation reaches around $80 \%-90 \%$ of the demand when the demand is far from the rest focus. From Equation 4, we have

$$
\frac{a_{s S}-a_{R F}}{d_{s S}-a_{R F}}=\frac{G_{\text {open }}}{1+G_{\text {open }}}
$$

so the observation that accommodation is around $80-90 \%$ of demand implies that $G_{\text {open }} /\left(1+G_{\text {open }}\right)$ is around $0.8-0.9$, and in turn that $\mathrm{G}_{\text {open }}$ must be in the range 4-9.

369 A property of any LTI system is that (after initial onset transients have died away) its response to a sinusoidal input is a sinusoidal output, with a gain and phase reflecting the transfer function of the system. Specifically, if the closed-loop transfer function is $H_{\text {closed }}(s)$, then if accommodative demand is a sinusoidal function of time, the accommodative response will also be a sinusoid with the same temporal frequency $f$. The amplitude of the response will be the amplitude of the demand multiplied by the gain at that frequency, $g(f)$, and the phase will be delayed by $\phi(f)$. We will use lower-case $g(f)$ to denote the gain of a system at a particular temporal frequency $f$, and upper-case $G=g(0)$ to denote the steady-state gain, as we did above for $G_{\text {open. }}$ According to a standard result of LTI theory, the gain and phase-delay of an LTI system at frequency $f$ can be obtained from the complex number represented by its transfer function $H(s)$ evaluated at $s=2 \pi j f$. The gain $g(f)$ is the magnitude of the complex number $H(2 \pi j f)$ and the phase-delay $\phi(f)$ is its phase.

Sometimes below for brevity we will refer to "the gain" of an LTI operator, without specifying a frequency. In this case, we mean its steady-state gain. For example, when we refer to "the gain" of a low-pass filter, we mean the ratio of its steady-state output to a constant input.

\section{Sensorimotor latencies: a problem for control}

387 These preliminaries out of the way, we now consider different possibilities for the contents of the block labelled Accommodative Control System in Figure 2. We begin by expanding this block as shown in Figure 5. We now explicitly include the rest focus signal discussed above. But critically, Figure 5 now also shows the system's latency, which we have divided into two parts. The first is an afferent-sensory latency, representing the time taken for information about the retinal image to travel up the optic nerve and for the brain to compute a signed estimate of 
393

394

395

396

397

398

399

400

401

402

defocus, for example using longitudinal chromatic aberration or higher-order aberrations. The second is an efferent-motor latency, representing the time taken for the resultant neural signal to travel from the Edinger-Westphal nucleus down the IIIrd cranial nerve and reach the ciliary muscle. These have been estimated as $T_{\mathrm{sens}} \sim 200 \mathrm{~ms}$ and $T_{\mathrm{mot}} \sim 100 \mathrm{~ms}$ respectively (Gamlin et al., 1994; Schor et al., 1999; D. Wilson, 1973). In Figure 5, these latencies are shown within the Accommodative Control System, i.e. the brain, but the model functioning is unchanged if, for example, part of the motor latency occurs at a neuromuscular junction in the eye, or indeed if both latencies are merged into a single block.

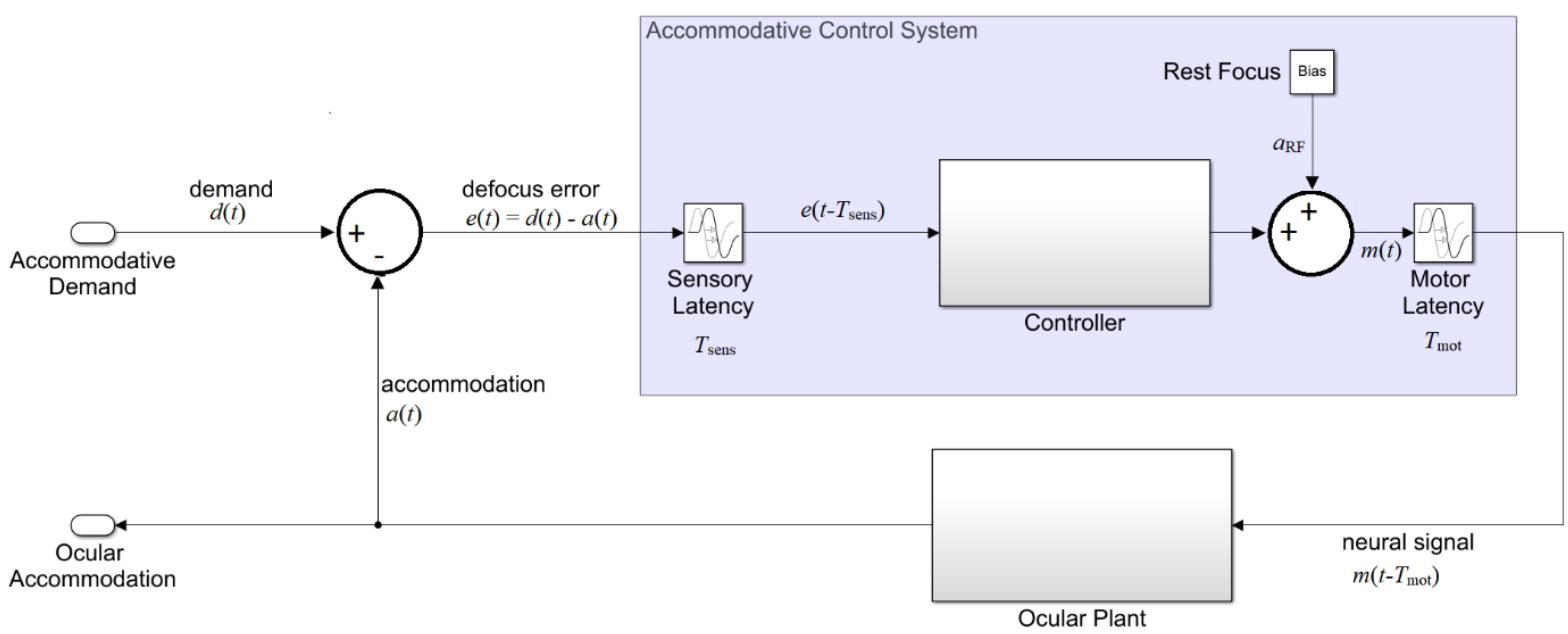

403

404

405

406

407

408

409

410

411

412

413

414

415

416

Figure 5. Expanding the conceptual model shown in Figure 2 so as to show the rest focus and sensorimotor latencies. This is the same circuit diagram, but the block labelled Accommodative Control System has here been expanded to explicitly show the constant bias signal accounting for the rest focus, and the latencies. There is a sensory latency $T_{\text {sens }}$ before the retinal defocus signal reaches the controller, and a further motor latency $T_{\text {mot }}$ before the neural signal reaches the plant.

Latencies are a potentially serious problem for any control system. In the block diagram shown in Figure 5, we can see that the defocus error only becomes available to the block marked Controller after the sensory latency. The controller therefore operates not on $e(t)$, but $e\left(t-T_{\text {sens }}\right)$ : the retinal defocus as it was a time $T_{\text {sens }}$ ago. This in turn reflects the accommodation due to the neural signal sent up to a time $T_{\text {sens }}+T_{\text {mot }}$ ago. Thus, the system suffers an overall latency of $T_{\text {lat }}$ $=T_{\text {sens }}+T_{\text {mot. }}$ This can easily lead to overshoots and "ringing": oscillations in accommodation as the system is driven beyond the correct value by the out-of-date error signal. 
417 Overshoots and ringing due to an out-of-date error signal would be seen with the response to

418 step changes in demand, but in fact the second-order dynamics already indicate that LTI models

419 do not suffice to account for the response to large step changes; accommodative control seems

420 to have special mechanisms for these which are beyond the scope of this paper (Bharadwaj \&

421 Schor, 2005, 2006; Schor \& Bharadwaj, 2005, 2004). However, an out-of-date error signal

422 would also affect the response to sinusoidal oscillations in demand which we are concentrating

423 on here.

425 Empirically, accommodation shows a low-pass response: gain is greatest in the steady-state, 426 and decreases monotonically with temporal frequency (Charman \& Heron, 2000; Krishnan et 427 al., 1973; Kruger \& Pola, 1986; Ohtsuka \& Sawa, 1997; Stark et al., 1965). However, it is 428 challenging to achieve this with the circuit diagram shown in Figure 5 and a Controller block 429 which is simply a PID controller. Because of the latency, the system can easily end up out of 430 phase, so that the changes in accommodation actually enhance the defocus rather than reducing 431 it, as intended. This shows up as resonances or local peaks in the gain function, making it nonmonotonic. This is not observed empirically. To avoid this, the controller must be more complex, as shown in Figure 6.

Overcoming latencies with a predictive control system: the Smith Predictor

436 The solution seems to be that the visual system actually bases its neural control not on the

437 currently available sensed value of retinal defocus, but on its internal prediction of the future 438 retinal defocus. That is, whereas in Figure 5 the controller operates on the sensed defocus, 439 which due to the sensory latency actually represents defocus as it was some time in the past, in 440 a predictive model the controller operates on the fpredicted future defocus (Smith, 1957). 441 Figure 6 shows how Figure 5 can be modified so that the input to the controller is predicted 442 future defocus. Defocus is the difference between the stimulus accommodative demand and the 443 ocular accommodation, so predicting future defocus requires a prediction both of demand and accommodation.

446 The brain is in principle able to predict accommodation perfectly up to future times less than 447 the motor latency, simply based on the signals it has already sent to the accommodative plant. 448 (Campbell \& Westheimer, 1960; Hung et al., 2002; Krishnan et al., 1973; Schor \& Bharadwaj, 449 2004; Stark et al., 1965; F. Sun et al., 1989) To do this, the visual system must effectively have 450 its own internal model of the ocular plant, represented by the Virtual Plant block in Figure 6. 
451 Such internal models are referred to as forward models in control systems theory. We assume that the motor latency $T_{\text {mot }}$ largely represents delays in transmitting the control signal from the brain to the eye. We assume that the virtual plant is located in the brain close to where the neural control signal is generated, and thus has access to this signal with negligible delay. Accordingly, the output of the virtual plant is predicted future accommodation, i.e. the value that ocular accommodation will have at a time $T_{\text {mot }}$ in advance of the present. We write this predicted future accommodation as $\hat{a}\left(t+T_{\mathrm{mot}}\right)$ : the predicted accommodation at a time $T_{\mathrm{mot}}$ in the future, where the circumflex indicates that this is an estimate of the future accommodation. Since the accommodation up to a time $T_{\text {mot }}$ into the future is controlled by neural signals already sent by the brain, this estimate can in principle be perfect. It should be affected only by noise, and by any inaccuracies in the virtual plant as a model of the ocular plant. In the model we present here, neither of these apply and so the prediction of future accommodation is indeed perfect.

466

467

468

469

470

471

472

473

474

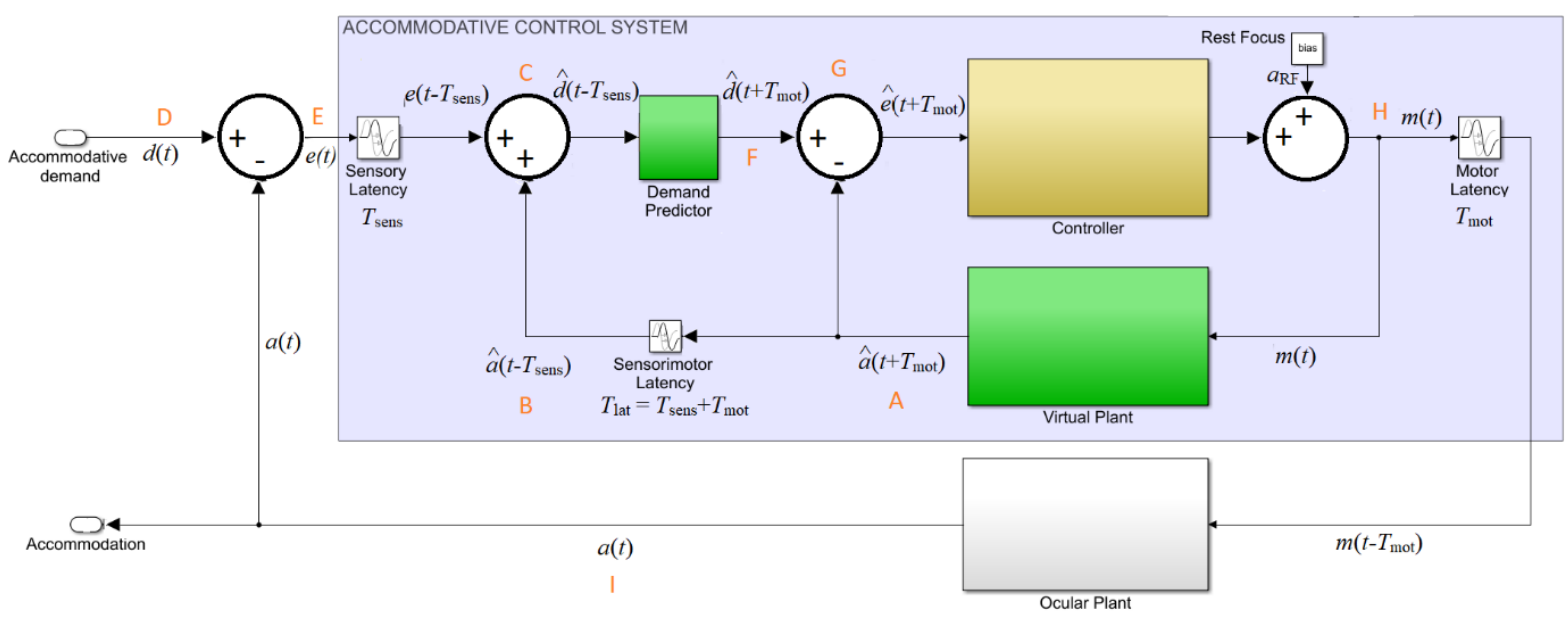

Figure 6. Predictive control. Compare with Figure 5: the Controller block has been replaced with a more complex system including two predictive blocks (green) as well as the original Controller block (yellow). The prediction helps avoid instability due to the sensorimotor latencies. To predict accommodation, the model includes a Virtual Plant block (forward model) to compute what accommodation will be a time $T_{\text {mot }}$ in the future, i.e. after the motor latency. If the forward model is accurate, this can in principle predict accommodation perfectly up to $t+T_{\text {mot, }}$ since accommodation is under the system's own control. To predict demand at time $T_{\text {mot }}$ into the future, the model uses a Demand Predictor block. This requires extrapolating demand at time $T_{\text {lat }}=T_{\text {sens }}+T_{\text {mot }}$ beyond the last available estimate. This is unlikely to be entirely accurate, since demand can reflect changes in the outside world, beyond the system's control. Orange labels indicate locations referred to in the text. 
476 Predicting stimulus demand is more challenging, since in general this reflects the motion of

477 objects in the outside world. Nevertheless, several studies (Campbell \& Westheimer, 1960;

478 Charman \& Heron, 2000; Krishnan et al., 1973; Phillips et al., 1972; Stark et al., 1965) have

479 suggested that the accommodation system, like other motor systems, may be capable of

480 predicting sufficiently regular input. For example, if the demand is a square wave, jumping

481 between two values with a constant period, accommodation develops a very short latency or

482 even changes in anticipation (Krishnan et al., 1973). How or whether this prediction is achieved

483 is beyond the scope of this paper; it may be performed by the cerebellum (Ohtsuka \& Sawa,

484 1997; Popa \& Ebner, 2019) or it may not actually occur (Águila-Carrasco \& Marín-Franch,

485 2021; Otero et al., 2019). The different possibilities can be modelled with the Demand

486 Predictor block (Figure 6). As will become clear below, the Demand Predictor block takes as

487 its input what demand is estimated to have been at time $T_{\text {sens }}$ in the past, $\hat{d}\left(t-T_{\text {sens }}\right)$, and gives 488 as output what it estimates demand will be at time $T_{\text {mot }}$ in the future, $\hat{d}\left(t+T_{\text {mot }}\right)$. That is, it 489 needs to extrapolate its input into the future by a time corresponding to the entire sensorimotor 490 latency, $T_{\text {lat }}=T_{\text {mot }}+T_{\text {sens }}$. In this paper, our model Demand Predictor block will simply pass its 491 input on unchanged, effectively assuming that the demand will stay at its current value. A future 492 model could incorporate a more elaborate form of prediction, e.g. taking account of stimulus 493 periodicity, but that is beyond the scope of this paper.

495 Having introduced the key elements of the predictive model - the virtual plant and the demand 496 predictor - we now discuss how it works. To help with this, we have annotated the signals in 497 Figure 6 and marked some reference points with orange letters. Let's start at A with the output 498 of the virtual plant. As we saw above, this represents the brain's prediction of what ocular 499 accommodation will be at time $T_{\text {mot }}$ in the future: $\hat{a}\left(t+T_{\text {mot }}\right)$. Our model brain uses this 500 predicted future accommodation in two ways. First (B), the model brain delays this predictedaccommodation signal by the total sensorimotor latency to obtain $\hat{a}\left(t-T_{\text {sens }}\right)$, an estimate of what the ocular accommodation was at a time $T_{\text {sens }}$ in the past. Thus, the predictive model actually uses an internal estimate of past accommodation as well as of future accommodation. The point of doing this is to match the latency of the defocus signal. The input to the whole system is accommodative demand, $d(t)$ (label D). In the eye (label E), the ocular accommodation $a(t)$ is optically subtracted from $d(t)$ to yield the error signal $e(\mathrm{t})$, the defocus at time $t$. Ideally, this is what the accommodation control should be based on, but due to the sensory latency $T_{\text {sens, }}$, the brain only has access to the delayed signal, $e\left(t-T_{\text {sens }}\right)$, representing the defocus at a time $T_{\text {sens }}$ 
ago. At the signal combination labelled $\mathrm{C}$, the brain adds its estimate of past accommodation, $\hat{a}\left(t-T_{\text {sens }}\right)$, back onto this delayed defocus signal $e\left(t-T_{\text {sens }}\right)$, in order to obtain an estimate of what the demand was at a time $T_{\text {sens }}$ in the past: $\hat{d}\left(t-T_{\text {sens }}\right)=e\left(t-T_{\text {sens }}\right)+\hat{a}\left(t-T_{\text {sens }}\right)$. This demand signal is fed into the Demand Predictor block, which uses it to make a guess at what the demand will be at a time $T_{\text {mot }}$ in the future: $\hat{d}\left(t+T_{\text {mot }}\right)$ (label F).

Now, the brain makes its second use of predicted future ocular accommodation, this time without applying any delay. At the signal combination labelled G, it subtracts the predicted accommodation $\hat{a}\left(t+T_{\text {mot }}\right)$ from the predicted demand $\hat{d}\left(t+T_{m o t}\right)$ to obtain the predicted future defocus: $\hat{e}\left(t+T_{\text {mot }}\right)=\hat{d}\left(t+T_{\text {mot }}\right)-\hat{a}\left(t+T_{\text {mot }}\right)$. This predicted future defocus is what is fed into the yellow Controller block and used to compute the neural control signal driving accommodation. It is this use of predicted future defocus which makes this a predictive model, as compared to the model shown in Figure 5.

As noted above, a constant bias is added on to the output of the controller, which accounts for the non-zero resting focus. We call the result $m(t)$ (label $\mathrm{H})$. This is the actual motor signal sent to the ocular plant, with a latency $T_{\text {mot, }}$ which results in the ocular accommodation $a(t)$ (label I). An efference copy of the same motor signal is also sent to be the input of the virtual plant. The output of the virtual plant is, of course, the predicted future accommodation that we began with (A), so we have now followed the signals around the whole of the inner loop.

In summary, then, although the input to the accommodative control system as a whole is the current optical defocus (Figure 2), in a predictive model the input to the accommodative controller itself is the predicted future defocus. With this modification, PID-type controllers can now work well and avoid the instabilities associated with an out-of-date error signal.

\section{Simplified representation of the predictive control system}

If the virtual plant is a perfect simulation of the physical plant, the predictive control system shown in Figure 6 is mathematically equivalent to the much simpler form shown in Figure 7. This form can appear confusing, because it shows accommodation being subtracted from the stimulus demand after the sensory latency (even though some of the sensory delay represents the optic nerve and cortical processing) and before the motor latency (even though that represents processes before accommodation). It is important to remember that apparently "non- 
causal" model diagrams like Figure 7 are a shorthand representing physiological predictive models like Figure 6, for the case where the virtual plant model is perfect. The reader is invited

544 to trace the signals around Figure 6 and Figure 7, and verify that provided $\hat{a}(\mathrm{t})=a(t)$, the same

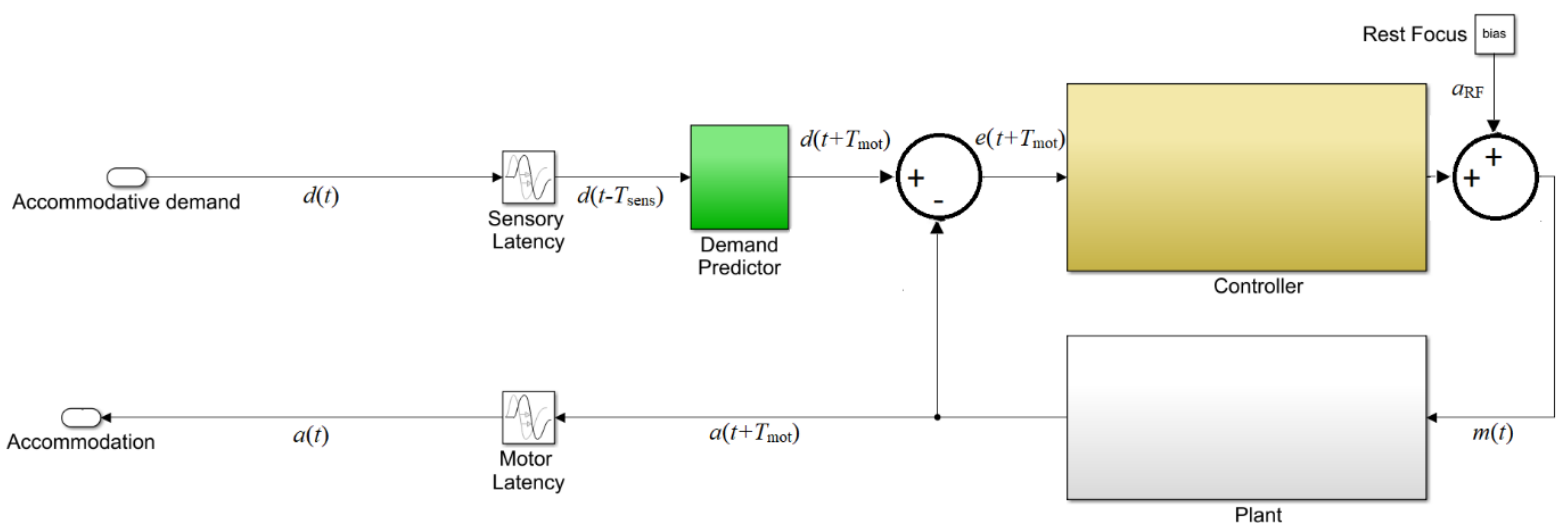

Figure 7. Simplified version of the model shown in Figure 6. This "non-causal" model structure is not physiological and cannot

be mapped onto "brain" and "eye" like the predictive physiological model in Figure 6. For example, here the single block

labelled "Plant" is used to represent both the physical plant in the eye and the virtual plant modelled in the brain. However, as shown by the annotated signals, it is mathematically equivalent to the physiological model in Figure 6, provided that the

556 The transfer function of this predictive model depends on the form of the demand predictor. We can distinguish two extreme cases: perfect vs "no change" prediction.

It is helpful to consider the unattainable ideal case in which the Demand Predictor block perfectly predicts the demand at a time $T_{\text {sens }}+T_{\text {mot }}$ beyond the last available information:

$$
\hat{d}\left(t+T_{m o t}\right)=d\left(t+T_{\text {mot }}\right)
$$

562 In this case, the closed-loop transfer function is

$$
H_{\text {closed }}^{\text {perfect }}(s)=\frac{P(s) C(s)}{P(s) C(s)+1}
$$

With perfect prediction, the sensorimotor latency has no effect at all on the gain or phase or indeed any other aspect of the response, since the perfect prediction annuls its effect. 
569 In many natural viewing situations, accommodative demand probably often changes rather 570 little over the timescale of $T_{\text {lat. }}$ Thus it may be relatively safe to adopt an attitude of "plus ça change, plus c'est la même chose" and assume that the demand at future time $t+T_{\text {mot }}$ will be the same as it was at the past time $t-T_{\text {sens }}$. In this case, the demand predictor block simply passes through its input as output:

$$
\hat{d}\left(t+T_{m o t}\right)=d\left(t-T_{\text {sens }}\right)
$$

In this case, the overall closed-loop transfer function is

$$
H_{\text {closed }}^{\text {nochange }}(s)=\frac{P(s) C(s) \exp \left(-s T_{\text {lat }}\right)}{1+P(s) C(s)}
$$

This is the simplest possible form of prediction: assuming things will stay the same as they are right now. And yet interestingly, the gain is exactly the same as for perfect prediction. Mathematically, this is because latency appears only in the numerator of Equation 10, and thus does not affect the magnitude of the complex transfer function, $\left|H_{\text {closed }}^{\text {nochange }}(j \omega)\right|$, which is what controls the gain at a given frequency (see Table 3). This means that the prediction of accommodation performed by the virtual plant suffices to avoid instability due to the latency, even if the "prediction" of demand is simply to assume it remains constant. Similarly, we can see that the phase of the "no change" transfer function is equal to the phase of the "perfect prediction" transfer function, plus a term $\omega T_{\text {lat }}$ reflecting the sensorimotor latency. In other words, the response of the "no change" predictive model to sinuosoids is exactly the same as the perfect predictive model, but delayed by the sensorimotor latency. This suggests that whereas predicting accommodation confers stability, the advantage of predicting demand is not stability, but shorter phase-delay and thus a more rapid response.

\section{A specific model of accommodative control}

596 So far we have deliberately kept the discussion very general, without committing to a particular choice of transfer function for either the ocular plant or the Controller block which converts defocus into a neural signal to the plant. In this section, we develop and justify a more specific 
model of accommodative control. We discuss plausible assumptions and constraints on both the forms of these transfer functions, and their particular parameters.

\section{Ocular plant}

The ocular plant can be regarded at least roughly as a first-order leaky integrator or low-pass temporal filter (A. P. A. Beers \& Van Der Heijde, 1994; A. P. Beers \& van der Heijde, 1996; Ejiri et al., 1969). We therefore model the transfer function of the plant as

$$
P(s)=\frac{1}{1+\tau_{\text {plant }} s}
$$

where empirically $\tau_{\text {plant }}$ is around 0.156 s for young eyes (Schor \& Bharadwaj, 2006). As noted above, we can assume without loss of generality that the steady-state gain of the plant is 1 .

\section{Controller}

We now come to a key decision: the choice of transfer function for the Controller, $C(s)$. As noted above, in industrial control systems, controllers typically have proportional, integral and derivative (PID) terms, with transfer functions which scale as constant, $1 / \mathrm{s}$ or s respectively.

We can rule out pure proportional control, since with $P(\mathrm{~s})$ as given in Equation 11, making $C(s)$ constant means that the system tracks rapid sinusoidal oscillations far better than human accommodation. For example, $C(s)=5$ results in a realistic steady-state gain of $83 \%$ (Equation 6 ), but the gain remains $>50 \%$ out to frequencies as high as $8 \mathrm{~Hz}$, far higher than observed (see Figure 8 below). Derivative terms do not affect steady-state error, but improve stability and avoid overshoot. They also enable rapid response to rapid changes. However, they can be problematic in the presence of noise. Previous work by Schor and Bharadwaj (Bharadwaj \& Schor, 2006; Schor \& Bharadwaj, 2006, 2004) suggests that the accommodative system has a distinct "pulse" mechanism for responding to sudden large changes in accommodation such as occur when we change from looking at a distant to a near object, which cannot be modelled by an LTI system and which are beyond the scope of this paper. Furthermore, many of the benefits of derivative control are already achieved by our use of a forward model to predict future demand. We therefore do not include a derivative term. This leaves us with the integral term. A pure integral controller has a transfer function proportional to $1 / \mathrm{s}$, and thus infinite gain at $\mathrm{s}=0$. This is desirable since it eliminates steady-state error, but as noted, the human accommodation does not seem to completely eliminate steady-state error. We can account for this by modelling the controller as a leaky integrator, following Krishnan and Stark (Krishnan \& Stark, 1975): 


$$
C(s)=\frac{G_{\text {fast }}}{1+s \tau_{\text {fast }}}
$$

where $\mathrm{G}_{\text {fast }}$ is the steady-state gain and $\tau_{\text {fast }}$ the time-constant. The subscript "fast" is to distinguish this from a slow integrator which we shall introduce below. A leaky integrator acts like a pure integral controller over short timescales $(s \tau>>1)$, and like a pure proportional controller over long timescales $(s \tau<<1)$, thus combining aspects of both. We noted above that accommodative lead/lag suggests the steady-state gain must be in the range 4-9. We somewhat arbitrarily chose $\mathrm{G}_{\text {fast }}=8$.

639

\section{Gain for sinusoidal input: sub-critical damping}

641 With both the plant and the controller being leaky integrators, and with a predictive control system, the closed-loop gain is that of a damped harmonic oscillator (Equation 21, Appendix). The behaviour of this system can be summarised by its natural frequency and damping coefficient $\zeta$, both of which depend on the parameters $G_{\text {fast }}, \tau_{\text {fast }}, \tau_{\text {plant }}$ (Equation 22 ). If the damping coefficient $\zeta$ is too low, the maximum gain is observed for a non-zero resonance frequency, and can even exceed 1. This does not agree with empirical observations of accommodative response to sinewaves, which is low-pass (Charman \& Heron, 2000; Kruger \& Pola, 1986; Ohtsuka \& Sawa, 1997; Stark et al., 1965); Figure 8A. This indicates that $\zeta$ is at least $1 / \sqrt{ } 2$, not far below critical damping $(\zeta=1)$ (Labhishetty \& Bobier, 2017). Saccades have a damping coefficient of around 0.7 (Bahill et al., 1975); systems with this value have minimum settling time, i.e. they reach and remain within $5 \%$ of their final value most rapidly. We show in the Appendix that obtaining $\zeta \sim 1 / \sqrt{ } 2$ for a system with $\mathrm{G}_{\text {fast }}>>1$ requires the time-constant of the fast controller to be

$$
\tau_{\text {fast }}=2 G_{\text {fast }} \tau_{\text {plant }}
$$

Thus, with $\tau_{\text {plant }}=0.156 \mathrm{~s}$ and $\mathrm{G}_{\text {fast }}=8, \tau_{\text {fast }}$ must be at least $2.5 \mathrm{~s}$.

660 Empirically, up to $\sim 1 \mathrm{~Hz}$ the phase delay of accommodation is very close to a linear function 661 of frequency, indicating a constant latency $\mathrm{T}_{\text {delay }}: \phi=2 \pi \mathrm{fT}_{\text {delay }}$ (Charman \& Heron, 2000; 
662 Heron et al., 1999; Kruger \& Pola, 1986; Ohtsuka \& Sawa, 1997; Wildt et al., 1974). The slope usually corresponds to a delay of $\sim 0.5 \mathrm{~s}$ (dashed lines in Figure $8 \mathrm{BC}$ ), though there is considerable variability between studies. Because this is close to the sensorimotor latency inferred from the response to step changes, it is often therefore assumed that this phase slope must represent the sensorimotor latency. However, this is not necessarily the case. First, the damped second-order system formed by the ocular plant and the neural control imposes delays in addition to the sensorimotor latencies. Second, in Equation 8 we saw that if the brain predicts demand perfectly - at least theoretically possible for a regular stimulus like a sinewave - then its phase delay becomes independent of the sensorimotor latency.

673 Thus, together the gain and phase response of accommodation to sinusoidal oscillations in 674 demand place quite tight constraints on the time-constant of the fast integrator, $\tau_{\text {fast, }}$ given that 675 the time-constant of the plant is a biomechanical given, and the gain of the fast integrator is 676 already quite tightly constrained by the observed lead/lag following a change in demand.

677 Figure 8 illustrates this by comparing the theoretical gain and phase with different values of $678 \tau_{\text {fast }}$ with empirical results from various subjects and studies. As noted, we can rule out $\tau_{\text {fast }}<$ $6792.5 \mathrm{~s}$ because the gain is then too high at high frequencies. The gain data is probably best 680 described by $\tau_{\text {fast }}=4 \mathrm{~s}$ (green lines), but this does not account for the phase data. $\tau_{\text {fast }}=4 \mathrm{~s}$ in the 681 perfect-prediction model gives phases which match empirical data up to around $0.5 \mathrm{~Hz}$, but at 682 higher frequencies, empirical phase continues to increase roughly linearly, implying a constant delay, whereas phase for the perfect prediction model asymptotes at $180^{\circ}$. Thus, we probably have to reject the perfect-prediction model (not surprising given its idealised nature). The nochange prediction model is qualitatively in much better agreement with the phase data, but then $\tau_{\text {fast }}=4 \mathrm{~s}$ predicts larger phases than are observed. The purple line shows the curve with minimum settling time, $\tau_{\text {fast }}=2.5 \mathrm{~s}$ which yields $\zeta \sim 1 / \sqrt{ } 2$. This is in reasonable agreement with both gain and phase data, assuming simple no-change demand prediction, and we therefore adopt this value in the rest of the paper.

690 

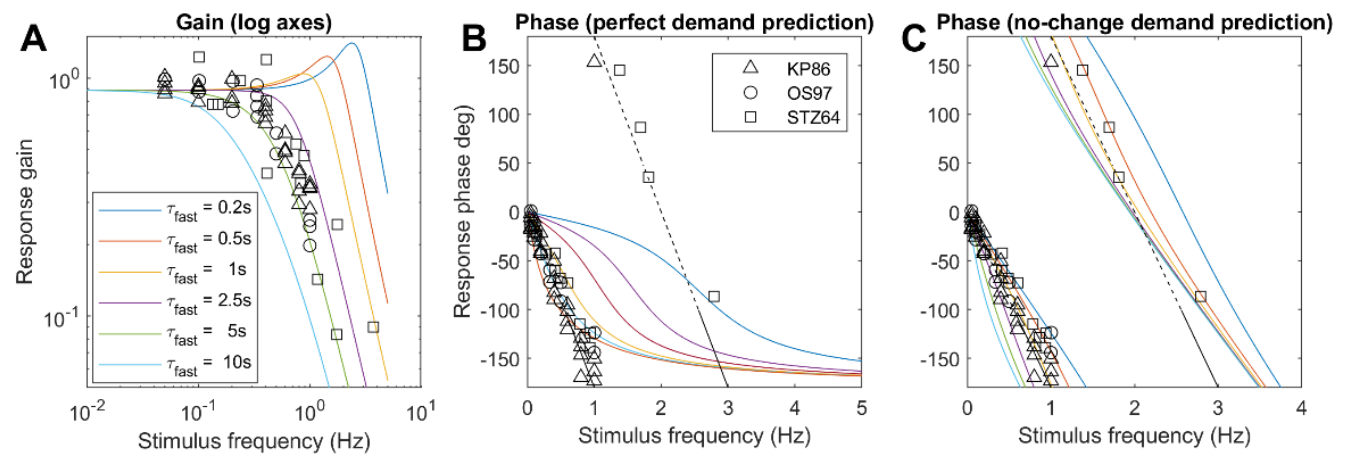

693 Figure 8. Constraints on the time-constant of the fast integrator. Coloured lines show the gain and phase predicted for a

694 predictive model with leaky-integral control, Table 3, with $P(s)$ given by Equation 11 with $\tau_{\text {plant }}=0.156 s$, and $C(s)$ given by

695 Equation 12 with $G_{\text {fast }}=8$ and different choices of $\tau_{\text {fast. }}$. The phase is shown for a model capable of predicting demand perfectly

696 (B), or for the "no-change" model which simply assumes demand will continue at its instantaneous value. Symbols show empirical results from Kruger and Pola (1986), Ohtsuka and Sawa (1997), and Stark et al (1965). Code to generate this figure is in Fig_TimeConstraints.m. The dashed line in the phase plots corresponds to a constant latency of $0.5 \mathrm{~s}$, close to what is observed.

Adaptation and dual control

702 Another distinctive feature of accommodation is that it adapts the open-loop decay time course to resting position after prolonged exposure to the same demand. This can be revealed by using pinholes to place the system in open-loop mode. As we have seen, in this situation, accommodation relaxes back to the resting focus. After short periods of stimulation, this happens rapidly, in a few seconds. However, after long periods of exposure to a particular demand, the relaxation happens over a much longer time period, sometimes several minutes. This cannot be accounted for with the leaky-integral control proposed so far. However, it can be explained by positing a dual control system in which a fast, or phasic, neural integrator controls changes in response amplitude and a slow, or tonic, neural integrator maintains the response amplitude (Khosroyani \& Hung, 2002; Schor, 1979a; Schor et al., 1986; F. C. Sun \& Stark, 1990).

714 The fast integrator is the one we have considered so far, which responds to error signals computed from negative feedback. The slow integrator responds to the activity of the fast neural integrator, and not directly to the error signal. As the name implies, the slow integrator

717 has a long time constant, which means that it has little effect on the response to rapid changes

718 in demand, so our previous discussion is not invalidated by its addition. With this arrangement, the transfer function of the Controller becomes 


$$
C(s)=\frac{G_{\text {fast }}}{s \tau_{\text {fast }}+1}\left(1+\frac{G_{\text {slow }}}{s \tau_{\text {slow }}+1}\right)
$$

The steady-state open-loop gain of the system is therefore

$$
G_{\text {open }}=G_{\text {fast }}\left(1+G_{\text {slow }}\right)
$$

Figure 9 shows the control system of Figure 5, modified to include this second, slow integrator, since it is easier to appreciate its operation in a non-predictive system. Suppose the system starts from rest, with demand and accommodation both equal to the rest focus, so that the defocus error and the outputs of the fast and slow integrators are both zero and the neural signal sent to the plant is simply the bias signal, maintaining it at the rest focus. Suppose the demand then makes a step change to a nearer value, $\mathrm{d}_{0}$. This in turn makes the defocus error non-zero, which begins to charge up the fast integrator. This increases the neural signal $m(t)$ above the bias value, altering accommodation so as to reduce the error. It also begins to charge up the slow integrator. Thus, over short timescales, the neural signal controlling accommodation is set mainly by the output of the fast integrator. However, over long timescales, the slow integrator takes over. The ratio of their steady-state contributions is equal to the gain of the slow integrator (Schor, 1979b; Schor et al., 1986); for example, with our value $\mathrm{G}_{\text {slow }}=5$, steadystate accommodation is $83 \%$ due to the slow integrator and $17 \%$ due to the fast integrator. Now suppose that pinholes are applied, making the defocus error zero regardless of accommodation. In this non-predictive model, after a delay corresponding to the sensory latency, the signal entering the fast integrator instantaneously drops to zero, and the fast integrator begins to discharge. As the fast integrator discharges, accommodation drops rapidly, with a decay time corresponding to $\tau_{\text {fast. }}$. When the signal from the fast integrator has dropped far enough, the slow integrator begins to discharges as well, resulting in a second, slower decay

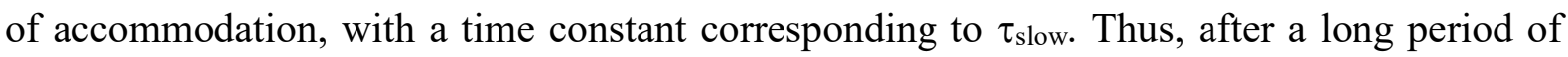
exposure, there is an initial rapid drop as the proportion of accommodation due to the fast integrator, initially $1 /\left(\mathrm{G}_{\text {slow }}+1\right)$, decays rapidly, but then a much longer decay as the dominant component due to the slow integrator decays slowly. 


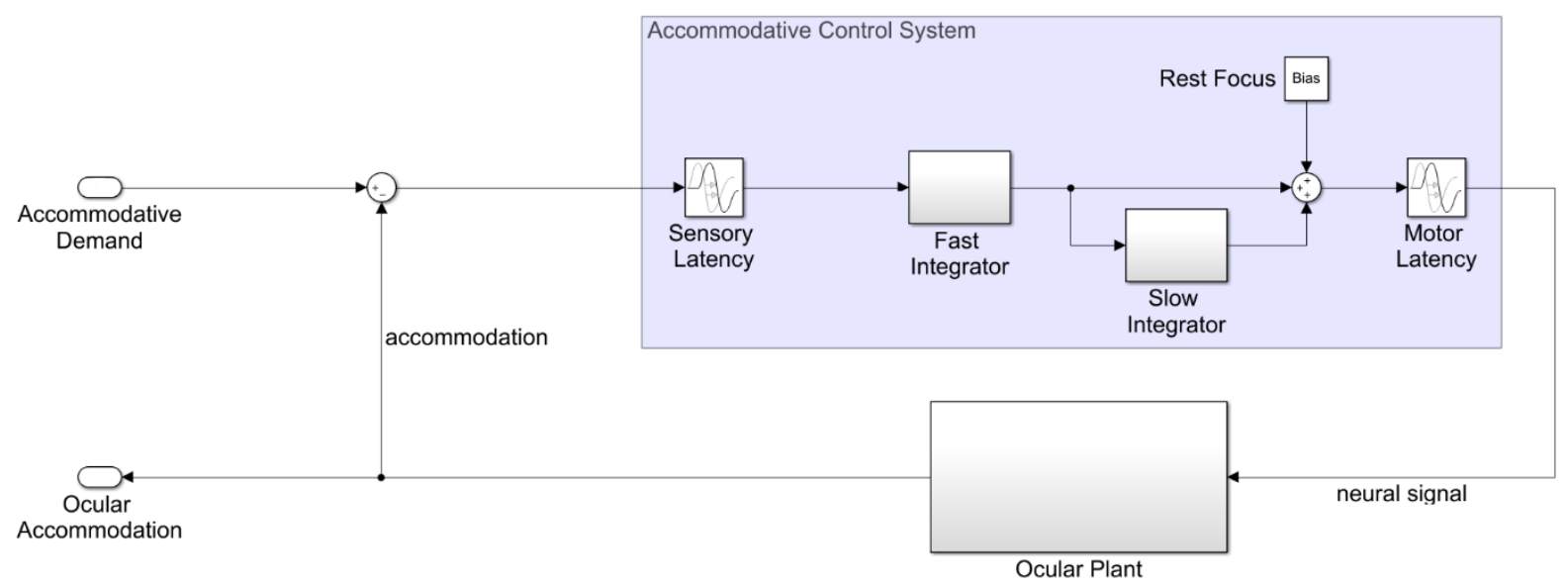

753

754

755

756

757

758

759

760

761

762

763

764

765

766

767

768

769

770

771

772

773

774

Figure 9. Non-predictive model incorporating dual (fast+slow) control. The slow integrator can be added to predictive models in the same way.

With predictive control, there is an additional subtlety. In such systems, the fast integrator is driven not by retinal defocus directly, but by the estimated future defocus (Figure 6). This does not immediately drop to zero when pinholes are applied. When the system is made open-loop by setting $d(t)=a(t)$, the input to the fast integrator becomes $a\left(t-T_{\text {sens }}\right)-a\left(t+T_{\mathrm{mot}}\right)$ for the nochange prediction model. This becomes zero once accommodation has stabilised, but is finite while it decays. When the gain of the slow integrator is sufficiently large, this small error input is enough to keep the slow integrator high. This in turn keeps accommodation high and thus sustains the error signal. Accommodation creeps slowly down to the rest focus with a timeconstant which, counter-intuitively, can be much longer than any of the three time-constants of the system: $\tau_{\text {plant }}, \tau_{\text {fast }}, \tau_{\text {slow. }}$ This effect is independent of exposure duration, so cannot account for the adaptation which the slow integrator was introduced to explain. To avoid this effect and obtain a clear difference between short and long exposure durations, we have found that $G_{\text {slow }}$ needs to be less than around 10 . Here, we have set $G_{\text {slow }}=5$.

The slow integrator also increases the overall steady-state gain and thus reduces the steadystate error. Using Equation 15 and Equation 6, the steady-state accommodative response is

$$
a_{s S}=a_{R F}+\frac{G_{\text {fast }}\left(1+G_{\text {slow }}\right)}{1+G_{\text {fast }}\left(1+G_{\text {slow }}\right)}\left(d_{s S}-a_{R F}\right)
$$


where with $G_{\text {fast }}=8, G_{\text {slow }}=5$ the gain term is 0.98 , compared to 0.89 with only the fast integrator. Thus, following a step-change in demand, the model response rises rapidly to around $90 \%$ of the demand, and then over the next tens of second rises more slowly to approach the demand exactly.

\section{Microfluctuations and noise}

Accommodation is subject to fluctuations, often called microfluctuations although they are actually quite substantial at around $\pm 0.5 \mathrm{D}$, exceeding the depth of field (Charman \& Heron, 1988, 2015). The source and purpose of these is unclear: they may represent neural noise, disturbances from the intraocular pulse, mechanical resonances within the ocular plant, deliberate attempts at "hunting" in order to find the best point of focus, and/or fluctuating input from the other influences on accommodation mentioned above (Charman \& Heron, 1988, 2015; Collins et al., 1995; Denieul, 1982; Gray et al., 1993b).

The power spectrum of open-loop accommodation is roughly a straight line on log-log axes (Campbell et al., 1959b; Campbell \& Westheimer, 1960; Stark et al., 1965), i.e. a power-law spectrum, $\mathrm{P}=1 / f^{\alpha}$. We model this by injecting white noise onto the defocus signal prior to input to the neural controllers (Figure 11). White noise has a flat power spectrum, but integration by the two integrators within the system (the neural controller and the plant) converts it to a powerlaw spectrum, with an approximately Brownian $\left(1 / \mathrm{f}^{2}\right)$ spectrum.

Noise has often been omitted from models of accommodative control, presumably with the rationale that once the correct noise-free response has been obtained, noise can always be added later to simulate microfluctuations. However, this approach is unwise, because noise in fact adds important constraints to the system. This is especially true with a predictive control system, which can easily end up amplifying noise in the open-loop condition. Referring to Figure 6, we see that a predictive control system actually contains not one but two feedback loops: one via the eyes, and one internal to the brain, incorporating the virtual plant. Operating in open-loop mode cuts the outer feedback loop, but leaves the internal feedback loop intact. Depending on the coefficients, internal noise can easily resonate within this loop, creating a situation where the power spectrum of open-loop accommodation has sharp peaks which do not occur in closed-loop mode, since the outer feedback loop suppresses them in its effort to keep the error zero. This is not observed empirically. The power of low frequencies does increase in open-loop mode (Charman \& Heron, 2015; Gray et al., 1993b), since without an 
808

813 Fortunately, we find that the values we have already derived are consistent with these data. A

error signal accommodation performs a random walk around the rest focus, whereas it is kept close to the demand in closed-loop mode. But we do not see an increase in the power of particular high-frequencies, as would occur if internal noise were resonating within the internal feedback loop.

more underdamped system - say $G_{\text {fast }}=15, \tau_{\text {fast }}=2$ s, which puts the damping coefficient $\zeta$ at 0.5 - does show unrealistic high-frequency resonances within the forward model feedback loop, but our sub-critically-damped parameters $G_{\text {fast }}=8, \tau_{\text {fast }}=2.5 \mathrm{~s}, \zeta=0.7$ already suppress the openloop resonance.

\section{Explaining the closed-loop resonance seen for high frequencies at low amplitudes}

In fact, several workers have found evidence for a resonance in closed-loop but not open-loop mode. The first evidence comes from microfluctuations during steady fixation. Several workers have found that the power-spectrum of closed-loop accommodation has a peak at around $2 \mathrm{~Hz}$ (Figure 10A). It is not always present, but when found is always more prominent in closedloop than open-loop accommodation. Although the location of this peak varies with heartrate, suggesting the pulse as a possible source interacting with blood volume of the ciliary body (Collins et al., 1995; Winn et al., 1990), the fact that it is higher in closed-loop conditions suggests that the source must be amplified by a neural resonance within the outer feedback loop.

Furthermore, the same resonance is assumed to be responsible for another puzzling observation, relating to gain with sinusoidal stimuli. In our discussion around Figure 8, we emphasised the lowpass nature of the gain response. This is true at high amplitudes, but for low-amplitude oscillations in demand, the curves become non-monotonic, with an increase in gain at around 2Hz (Figure 10B). Ockham's Razor suggests this reflects the same closed-loop resonance causing the $\sim 2 \mathrm{~Hz}$ peak in microfluctuations. However, the dependence on amplitude indicates that this resonance must be caused by a nonlinear mechanism, since for a linear system gain is independent of stimulus amplitude. 

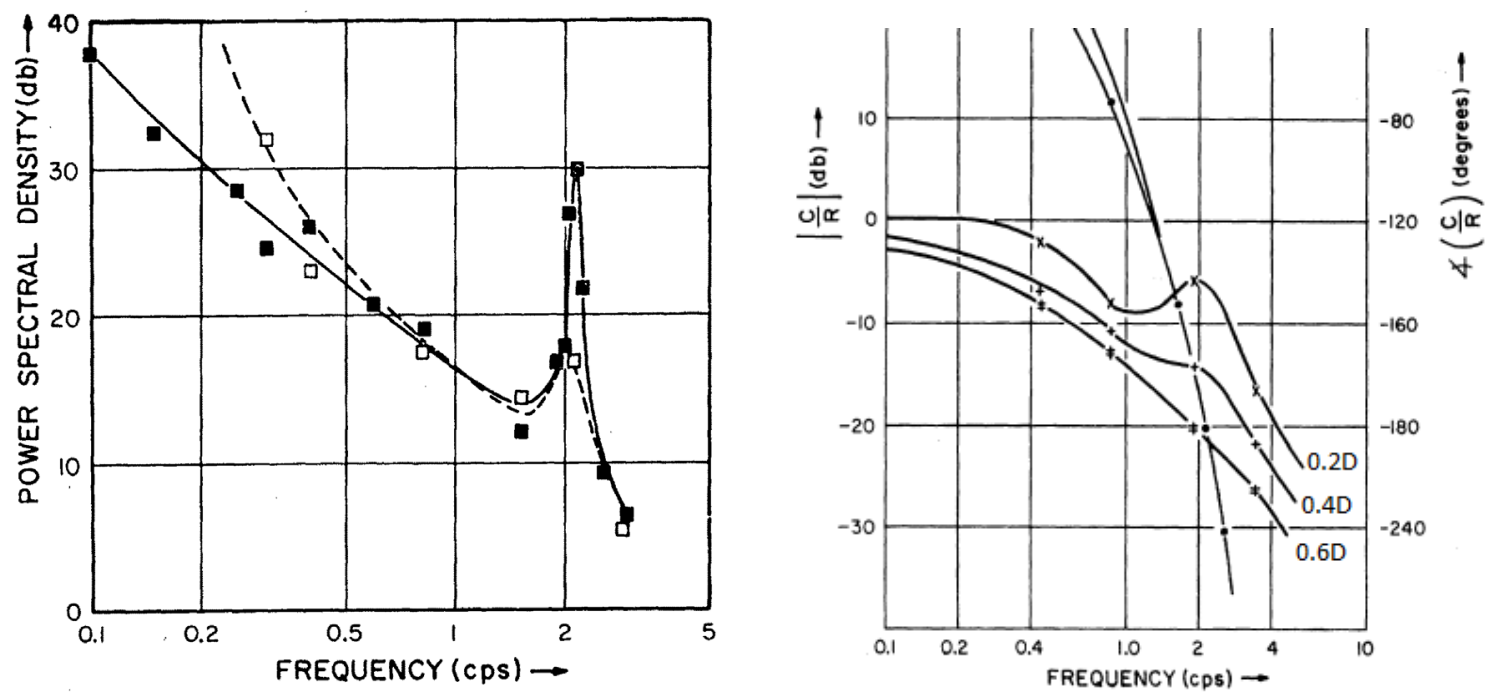

Figure 10. Evidence for a resonance at around $2 \mathrm{~Hz}$ in accommodative control. (A) Figure 5 from Stark et al 1965 (Stark et al., 1965), replotting empirical results from Campbell et al 1959 (Campbell et al., 1959b), showing the power spectrum of accommodation under closed-loop (solid) and open-loop (pinhole, dashed) conditions. (B) Empirical results from Figure 4 of Stark et al 1965, showing gain for sinusoidal oscillations of three different amplitudes $(0.2 D, 0.4 D, 0.6 D)$. Gain is expressed in

846 Resonances observed in closed- but not open-loop mode immediately suggest a control system

847 lacking the predictor we have argued for so far. Non-predictive control is prone to closed-loop instabilities in systems with latencies, like accommodation. This occurs in the outer feedback loop via the eye, when the accommodation change designed to null out defocus arrives out of phase due to the latency and ends up enhancing the defocus which cause it. Predictive control avoids these closed-loop instabilities, but if the prediction is imperfect, it can be vulnerable to open-loop resonances due a similar effect occurring via the internal feedback loop driven by the efference copy. (For a mathematical justification of these statements, see the Appendix, specifically the discussion around Equation 17, Equation 18 and Equation 20.)

856 Thus to explain both the power spectrum of microfluctuations, and the non-linear resonance in 857 the response to sinusoidal demand, we postulate an additional signal controlling 858 accommodation. This is proportional to small amplitudes of the current defocus, not the estimated future defocus, and is thus not predictive. (This signal is, however, included within

860 the efferent copy used to estimate future defocus within the predictive control system.) Because 861 this signal is non-predictive, it is prone to closed-loop instabilities. But for the same reason, it avoids open-loop resonance which can occur within a predictive system. 
863 To prevent the closed-loop instabilities from catastrophically destabilizing the response, we

864 clip this non-predictive signal at a low value, set to $0.15 \mathrm{D}$ in our model (i.e. signals larger than

$8650.15 \mathrm{D}$ in magnitude are set to $\pm 0.15 \mathrm{D}$ depending on their sign). This saturating value is chosen

866 simply because it gives a reasonable match to empirical results. It is low enough to ensure that

867 the signal does not change the behavior of the model in response to large changes in defocus.

868 However, it is large enough that the signal still produces a visible high-frequency peak in the

869 power spectrum of closed-loop microfluctuations and a high-frequency resonance in the

870 response to low-amplitude sinusoids (see Results).

871 This non-predictive saturating signal has other interesting effects on accommodation. Notably,

872 it facilitates a rapid response to small step stimuli, because non-predictive proportional signals

873 tend to react faster than predictive integral signals. For example, suppose demand suddenly

874 increases by $0.1 \mathrm{D}$, causing an $0.1 \mathrm{D}$ step-change in defocus. The non-predictive proportional

875 control signal, with unit gain, requests the full 0.1D increase in accommodation. The fast

876 integrator begins responding at the same time, but due to its integral nature, its response ramps

877 up more gradually. Furthermore, because the non-predictive proportional signal uses the

878 current sensed defocus, rather than the predicted future defocus, it stays requesting the full

$8790.1 \mathrm{D}$ for at least $0.3 \mathrm{~s}$, until the sensorimotor latency has elapsed and the ocular plant starts to

880 respond and thus reduces the sensed defocus. In contrast, input to the fast integrator is estimated

881 future defocus, which begins to fall immediately based on the requested change to 882 accommodation (the predictive control system assumes that demand will stay at the new value,

883 but it predicts that defocus will fall because of the predicted accommodative response). So, the

884 input to the fast integrator begins to fall immediately from its initial peak of $0.1 \mathrm{D}$, whereas the

885 input to the proportional controller stays at 0.1D until the sensorimotor latency has elapsed.

886 Thus for small step-changes in defocus, the non-predictive proportional signal enables a larger,

887 faster response. However, the saturation means that its effect is limited to small changes, with

888 the predictive-integral control dominating the response to large changes. Dynamics of larger 889 step responses are controlled with a pulse signal (Bharadwaj \& Schor, 2005, 2006; Schor \& 890 Bharadwaj, 2006, 2004) that will be added to this model in a subsequent paper.

893 In principle, small enough changes in defocus that, given the eye's optics, produce no 894 significant change in the retinal image cannot drive accommodation. The smallest change in 
895 defocus which produces a detectable change in accommodation is referred to as objective depth

896 of focus. This is typically much smaller than the subjective depth of focus, i.e. the smallest

897 change in defocus which produces a perceptible change in image quality (Kotulak \& Schor,

898 1986a; Udlam et al., 1968; Yao et al., 2010). Depth of focus is often modelled as a deadzone

899 (e.g. (Schor, 1979b)): the defocus signal is set to zero unless it exceeds some threshold value

900 corresponding to the objective depth of focus, say $0.2 \mathrm{D}$. However, this approach has a number

901 of drawbacks:

902 (i) It can result in unrealistic jumps, where a small change in demand pushes the defocus above 903 the threshold and thus elicits a disproportionately large response.

904 (ii) It produces a hysteresis effect, whereby accommodative lead and lag can depend on how

905 the demand is approached. For example, with a threshold of $0.2 \mathrm{D}$, if the demand steps up from

$9061 \mathrm{D}$ to $2 \mathrm{D}$, the effective defocus becomes zero once accommodation reaches $1.8 \mathrm{D}$, so we get a

907 lag. But if demand steps down from 3D to 2D, effective defocus becomes zero once

908 accommodation reaches 2.2D, so we get a lead. This hysteresis is not typically observed, except

909 with extremely blurred images (Heath, 1956a) .

910 (iii) It reduces the gain of the response to low-amplitude oscillations. For example, consider a

911 slow oscillation ranging between 1D and 3D. Assume for simplicity that the closed-loop gain

912 of the system is 1 , so that in the absence of a deadzone, the response would track demand

913 exactly. With a deadzone clipped at $0.2 \mathrm{D}$, the response would range from $1.2 \mathrm{D}$ to $2.8 \mathrm{D}$,

914 reducing the gain to 0.8 . With a lower-amplitude oscillation where demand ranged from 1.5D

915 to $2.5 \mathrm{D}$, the response would range from $1.7 \mathrm{D}$ to $2.3 \mathrm{D}$, making the gain 0.6 . With a still lower-

916 amplitude demand ranging from 1.7D to 2.3D, response would range from 1.9D to 2.1D,

917 making the gain 0.3 . Yet this decrease in gain with decreasing amplitude is not observed. In

918 fact, accommodative gain tends to be smallest for high amplitudes, not for low amplitudes

919 (Stark et al., 1965, p. 196).

920

921 For all these reasons, we have chosen not to include a dedicated defocus deadzone in our model.

922 The objective depth of focus is adequately accounted for by the white noise we have added to the defocus signal, which effectively swamps small changes.

924

925

926 


\section{Simulink implementation and summary of the model}

928 Figure 11 shows the complete model as it appears in our Matlab Simulink implementation,

929 incorporating all the elements discussed above. The model has two inputs: (1) "demand",

930 accommodative demand in diopters, and (2) "pinhole", which conveys whether the eye is

931 currently viewing through a pinhole or not. If pinholes are present, the defocus signal is set to

932 zero; otherwise it is set to demand minus accommodation. The defocus signal has white noise

933 added to it and is delayed by the sensory latency before reaching the "brain" module.

934

935 Here, four signals are combined to produce a neural signal which is delayed by the motor

936 latency before reaching the ocular plant. From top to bottom, these four signals are: (1) the

937 constant bias signal, which sets the rest focus; (2) the proportional signal, which is simply the

938 noisy defocus signal clipped at $\pm 0.15 \mathrm{D}$; (3) the signal from the fast integrator, which is driven

939 by the estimated future defocus; (4) the signal from the slow integrator, which is driven by the

940 fast integrator. One final detail not mentioned so far is that the neural signal is thresholded at

941 zero to ensure it is positive. This is visible in the diagram as the "saturation" block on the far

942 right, immediately after the four signals are combined. This accounts for the fact that the ciliary

943 muscle can only be commanded to contract, making the lens more convergent, or allowed to

944 relax. Negative values would effectively command the ocular lens to adopt a divergent form,

945 which is physically impossible.

946

947 As well as being sent down cranial nerve III to the eye, an efferent copy of the neural signal is

948 directed to a virtual plant within the brain, which predicts the future accommodation. This in

949 turn is used to estimate the future defocus which drives the fast integrator. For completeness,

950 we have included a block labelled "Demand Predictor", although in the current instantiation of the model, this simply passes its input through unchanged. 


\section{Simulation details}

Figure 11. Simulink block diagram of our final model, incorporating all the features discussed in the paper. The Simulink model has two inputs: (1) demand, and (2) whether or not the eye is viewing through a pinhole. It has one output: accommodation 
976 The simulation shows onset transients at its start point, as the integrators settle. In all cases, we therefore discarded the first few seconds of simulation time in order to exclude these transients.

\section{Results}

980 The different elements of this model were motivated by different observations - the gain and

981 phase to sinusoids; adaptation; power spectra of microfluctuations so on. Components such as

982 the fast and slow integrator and the virtual plant have been proposed before for the

983 accommodation step response (Schor \& Bharadwaj, 2005), but to our knowledge never tested 984 in combination for pursuit sinusoidal tracking (Schor \& Kotulak, 1986) or adaptation (Schor, 985 1979b), or with white noise and the feeding through of a clipped signal proportional to the 986 current defocus. This combination is to our knowledge a novel contribution. We now 987 demonstrate that this unified model can reproduce each of the observations that motivated its 988 different components.

989

\section{Response to sinusoidal demand}

991 Figure 12 shows the gain and phase of the model (heavy black line), compared with results 992 from human subjects digitised from (Kruger \& Pola, 1986; Ohtsuka \& Sawa, 1997). This is of course similar to results already shown in Figure 8, but whereas those curves were obtained

994 from mathematical formulae for a leaky integrator in a predictive control system, Figure 12 is 995 obtained via Simulink simulation of the full four-signal model with noise. There is reasonable 996 agreement in gain (Figure 12AB); both humans and model are low-pass. The main quantitative 997 disagreement is that the "knee", where the gain drops rapidly, typically occurs around $0.4 \mathrm{~Hz}$ 998 in humans and slightly later, around $0.6 \mathrm{~Hz}$, in the model. There is also good agreement in phase 999 (Figure 12C). For comparison, the dashed black line shows the phase which would be obtained 1000 for a model with perfect demand prediction. As we showed above, this can be obtained from 1001 the phase of our model with "no change" demand prediction by subtracting the sensorimotor 1002 latency: $\phi_{\text {perfect }}=\phi_{\text {nochange }}-360 f T_{\text {sens }}$. Interestingly, the phase function of most human subjects 1003 agrees better with that of the no-change model rather than the perfect model, suggesting that these subjects had little ability to predict the oscillatory demand. 

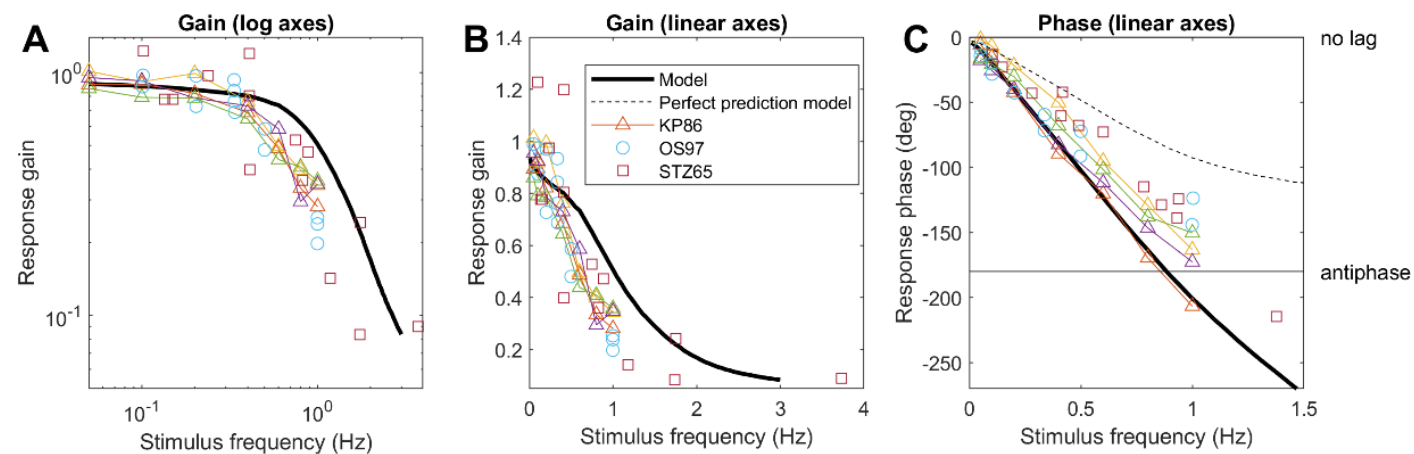

Figure 12. Gain and phase of the model response to sinusoidal demand, compared to empirical results. A,B: Gain plotted on

linear and log axes. C: Phase plotted on linear axes. The heavy black line is the response of the model in Figure 11 with the parameters given in Table 2. The dashed black phase line shows the phase which would be obtained by a model capable of perfectly predicting the sinusoidal oscillation in demand. Triangles show empirical results for four human subjects, digitised from Kruger and Pola (Kruger \& Pola, 1986), using the data with white light and defocus cue only. Circles are for a further four subjects, digitised from Ohtsuka and Sawa (Ohtsuka \& Sawa, 1997), using only their control subjects. In (Kruger \& Pola, 1986) and in the model, the demand oscillated between $1 D$ and 3D, i.e. the amplitude of the sinusoid was 1D and its mean value was 2D. In (Ohtsuka \& Sawa, 1997), the amplitude was 1.5D and its mean value is not stated. Code to generate this figure is in Fig_CompareGainPhase.m. Run_Sine.m must be run first to generate the model data.

1019 Figure 12 was for sinusoidal demand oscillations with an amplitude of 1D. Of course, the gain and phase of a linear system are independent of amplitude. However, our model is nonlinear due to the saturation of the non-predictive proportional signal. Figure 13 shows the gain and phase in the same format as Figure 12, but for different amplitudes of oscillation around a 2D baseline. The green lines are for the 1D amplitude shown in Figure 12, but for lower amplitudes the gain and phase start to deviate significantly from these results. Most strikingly, there is a resonance at $1.2 \mathrm{~Hz}$ where the gain actually goes above 1 for the smallest oscillations $( \pm 0.1 \mathrm{D})$. This represents the instability caused by the non-predictive proportional signal. Since this signal is clipped at $\pm 0.15 \mathrm{D}$, it has a significant effect only for low-amplitude oscillations.
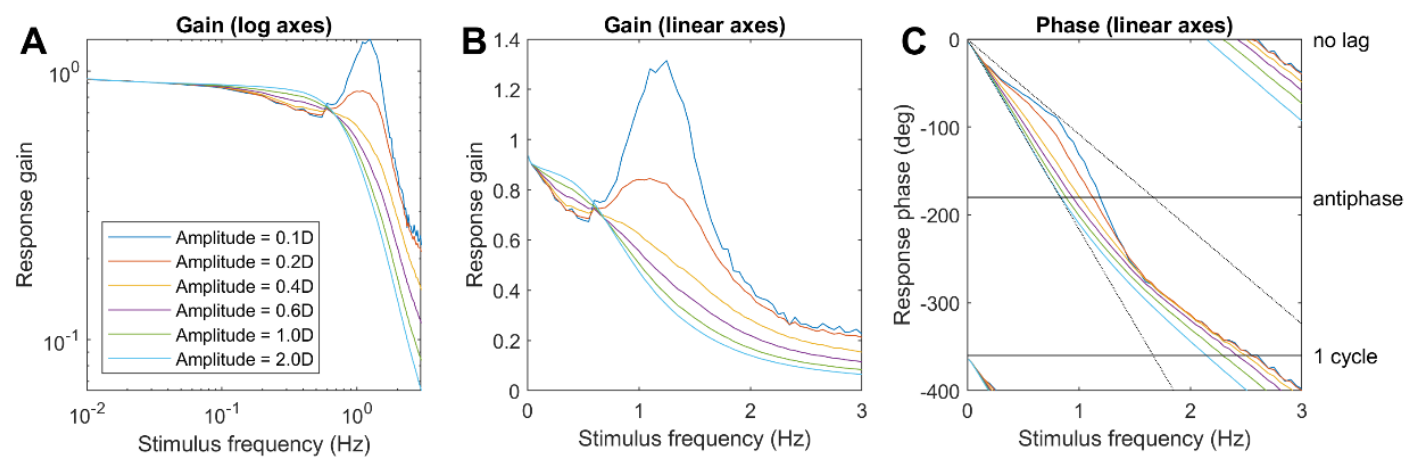
Figure 13. Model gain and phase as a function of amplitude. The green curves (1D) are what was shown in Figure 12, but we see that the behaviour at low amplitudes is quite different, with a resonance at $1.2 \mathrm{~Hz}$. Code to generate this figure is in Fig_Sine.m. Run_Sine.m must be run first to generate the model data.

This effect is qualitatively in agreement with the low-frequency resonance reported by Stark et al. (Stark et al., 1965), which led them to conclude that human accommodative control must include a nonlinearity. Digitized data from (Stark et al., 1965) is replotted in Figure 14, along with the response of the model. The model does not reproduce the strong dip in gain at $0.8 \mathrm{~Hz}$ for an amplitude of $0.3 \mathrm{D}$, but apart from that, the agreement is quite good. In particular, it accounts for the key observation that gain is quite high, around 0.5 , for $0.3 \mathrm{D}$-amplitude oscillations at around $2 \mathrm{~Hz}$, whereas gain is much lower, around 0.1 , for higher amplitude oscillations at this frequency.
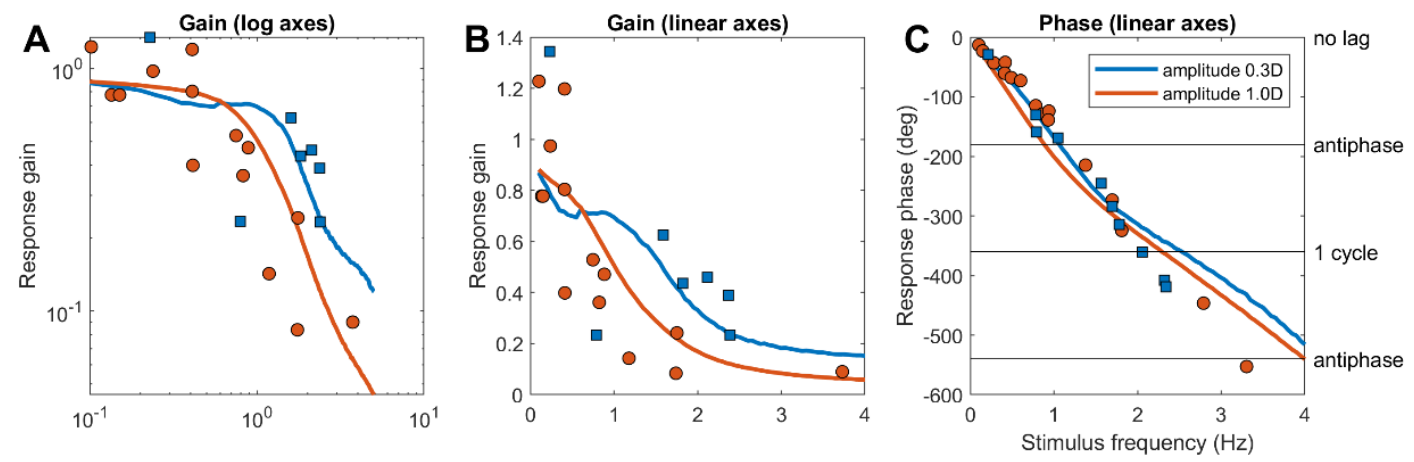

Figure 14. Symbols are digitised data from Figure 3 of Stark, Takahashi \& Zames (Stark et al., 1965). These are measured gain and phase for one subject, for amplitudes of $0.3 D$ (blue) and 1D (orange). The curves are model gains and phase for these amplitudes, about a baseline of 2D. Code to generate this figure is in Fig_StarkTakahashiZames.m. Run_StarkTakahashiZames.m must be run first to generate the model data.

1052 Figure 13C and Figure 14C showed that the phase of the response relative to demand increases 1053 with frequency, reaching 180 degrees at a frequency of around $1 \mathrm{~Hz}$. When this occurs, the 1054 demand and response are in antiphase, and the error is greater than the stimulus. Interestingly, 1055 if the response gain were zero, then the error for the 180 deg phase delay would be smaller than if the gain were 1.0. It is therefore of interest to ask how the gain and phase changes affect defocus error for demand oscillations of different amplitude and frequency. We quantify this using the mean absolute defocus error. The defocus error is the difference between demand and 
accommodation at any time; absolute defocus error is the rectified version of this waveform, and mean absolute defocus is the average value of this over time: $\langle|d(t)-a(t)|\rangle$, where $d(t)=D_{\text {mean }}+D_{\text {amp }}(\sin 2 \pi f t)$.

1063 The heavy curves in Figure 15A show how mean absolute defocus error varies with amplitude and frequency of sinusoidal demand. In each case, the peak error is just below $1 \mathrm{~Hz}$, when the response is $180^{\circ}$ out of phase with the demand (Figure 13C). The error increases with demand amplitude, even though for frequencies below the peak, the gain (i.e. the ratio of response to demand) is closer to 1 for larger amplitudes (Figure 13AB).

The aim of accommodative control is to track demand so as to minimize defocus error, but the phase-delay means that for sufficiently high frequencies, this aim would be better achieved by simply keeping accommodation fixed at the mean demand, i.e. by having a response gain of 0 , rather than attempting to track oscillations in demand about this baseline. The dashed lines in Figure 15A shows this zero-gain tracking error, i.e. the mean absolute defocus error which would be achieved if accommodation stayed at the steady-state value elicited by the mean demand $\left(D_{\text {mean }}=2 \mathrm{D}\right.$ in this example). Because the amplitude of zero gain tracking error depends only on the input amplitude, the error is independent of temporal frequency of the sine input. Since the static accommodative lag is small, the zero-gain steadystate response is also close to 2D. So the mean zero-gain error is approximately the average value of $\left|D_{a m p}(\sin 2 \pi f t)\right|$, or $2 \mathrm{D}_{\mathrm{amp}} / \pi$, where $\mathrm{D}_{\mathrm{amp}}$ is the amplitude of the demand oscillations about the $2 \mathrm{D}$ baseline.

We define the limiting tracking frequency to be the frequency at which the actual gain and phase-delay of the accommodative response produces the same error as would be achieved with zero gain. This is where the zero-gain tracking error is first equal to the actual error, marked with a cross $\mathrm{x}$ in Figure 15A. For frequencies lower than this limit, the oscillation in accommodative response is helpful, i.e. it tracks the oscillations in demand with a phase delay low enough to reduce the mean defocus error below the zero-gain tracking error. However for frequencies above the limit marked with a cross, the oscillatory response is out of phase and ends up making mean defocus error larger than if accommodation simply remained constant at the baseline value. 
bioRxiv preprint doi: https://doi org/10.1101/2021.12.09.471909; this version posted December 10, 2021. The copyright holder for this preprint (which was not certified by peer review) is the author/funder, who has granted bioRxiv a license to display the preprint in perpetuity. It is made available under aCC-BY 4.0 International license. signal, this limiting-tracking frequency depends on amplitude, as shown in Figure 15B. For

1094 large-amplitude oscillations in demand, accommodation can track only up to around $0.4 \mathrm{~Hz}$.

1095 We saw above that the non-predictive proportional signal enables a more rapid response to 1096 small changes. This is shown in Figure 15B by the increase in limiting tracking frequency for 1097 low-amplitude oscillations.

1099 Using the result that perfect demand prediction would reduce the phase by the sensorimotor 1100 latency, we can also infer what these curves would be for a model with perfect demand 1101 prediction but with the same leaky-integral controller. These are shown with the light curves 1102 in Figure 15AB. Perfect demand prediction does reduce the error and increase the limiting 1103 tracking frequency, but not dramatically, because of limits imposed by the time constant of the 1104 plant and the fast integrator.

A

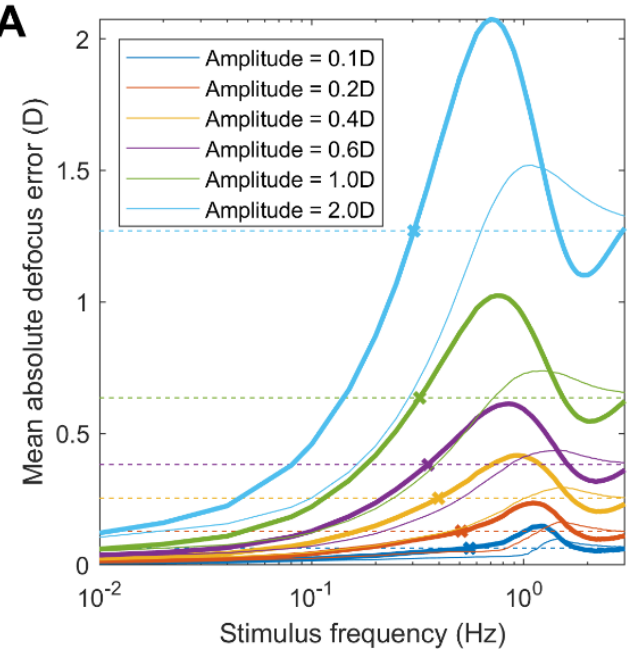

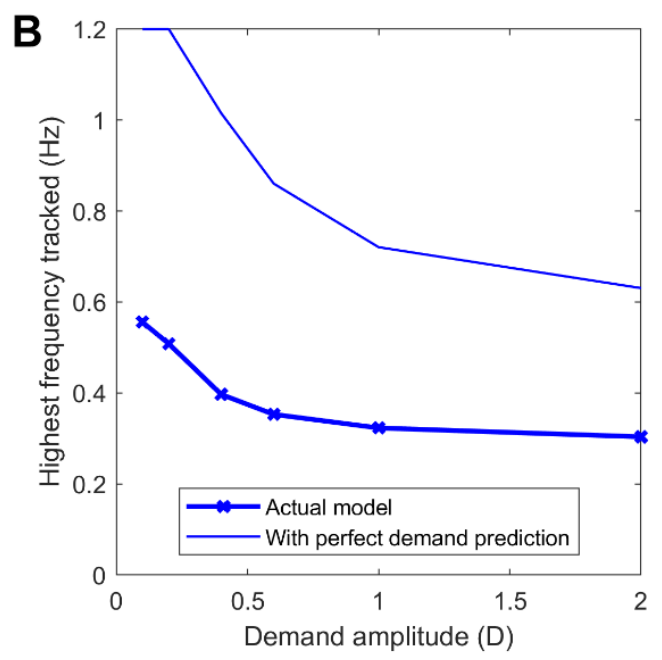

1106 Figure 15. (A) Mean absolute defocus error for sinusoidal demand oscillations of different frequencies and amplitudes about 1107 a 2D baseline. The heavy curves show $|d(t)-a(t)|$ for our model with its observed gain and phase; the light curves are those 1108 inferred for a model with perfect demand prediction. The dashed lines show the expected high-frequency limit, i.e. the mean 1109 defocus error if the demand oscillated but the response stayed at the steady-state value elicited by the mean demand, and 1110 the crosses indicate where this is first less than the error with tracking. The crosses mark where this crosses the mean defocus 1111 error. We take this as an indication of the highest frequency which can be successfully tracked at this amplitude. (B) Tracking

1112 frequency limit as a function of amplitude, for the actual model (heavy line, crosses) and for a model with perfect demand 1113 prediction (upper light line). Code to generate this figure is in Fig_Sine.m. Run_Sine.m must be run first to generate the model 1114 data. 
1117 Figure 16A shows example closed- and open-loop accommodation traces recorded from the

1118 model over the course of 5 minutes. The red trace is for closed-loop viewing of a stimulus at

1119 1D (red dashed line). Accommodation thus fluctuates around a value a little over 1D, reflecting 1120 the accommodative lead for a stimulus nearer than the rest focus, here 1.4D. The fluctuations 1121 span a range of around $0.1 \mathrm{D}( \pm 2 \mathrm{SD})$. The $\mathrm{SD}$ is $0.03 \mathrm{D}$, which is small compared to the SD of 1122 human microfluctuations (0.1-0.3D, (Charman \& Heron, 1988, 2015; Gambra et al., 2009)). 1123 The power spectrum, Figure 16D, has a prominent peak at around 1.5Hz. This periodic 1124 structure is clearly visible in the 10s except from the trace shown in Figure 16B.

1126 The blue trace is for open-loop viewing, e.g. through pinholes. Now, the response wanders 1127 around the rest focus, 1.4D (dashed blue line). However, because the bias is constant rather 1128 than scaling with the difference between accommodation and rest focus, the excursions are 1129 much wider. This is visible in the power spectrum, Figure 16D, where the power continues to 1130 rise as frequency reduces.

1132 Figure 16B shows a 10s excerpt from the trace in Figure 16A, for comparison with the example 1133 empirical data in Figure 16C, digitized from (Gray et al., 1993a). Although the amplitude of 1134 the microfluctuations is larger in the human observer, the same qualitative features are visible: 1135 closed-loop mode showing strong periodic structure at around $2 \mathrm{~Hz}$, open-loop mode showing 1136 much larger low-frequency fluctuations. Figure 16E shows the closed- and open-loop power 1137 spectra for a human observer, digitized from (Campbell et al., 1959b), for comparison with 1138 Figure 16D.

1140 The presence of this relatively large, $1-2 \mathrm{~Hz}$ periodic component in the closed-loop 1141 microfluctuations may aid accommodative control, for example by "hunting" for the point of 1142 optimal focus (Kotulak \& Schor, 1986c). Thus, this could be a reason why the postulated non1143 predictive proportional signal is beneficial for accommodative control. 

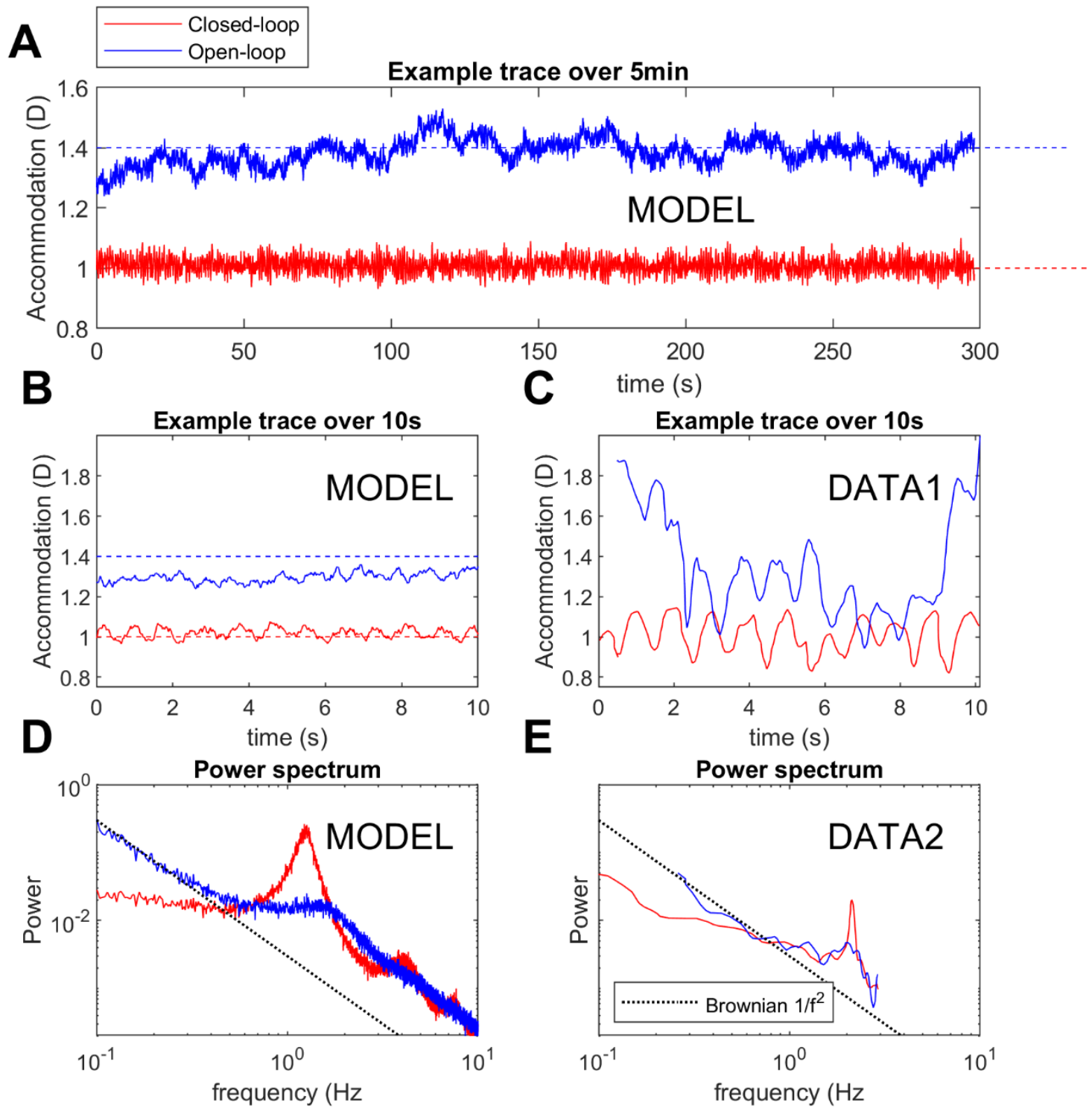

E

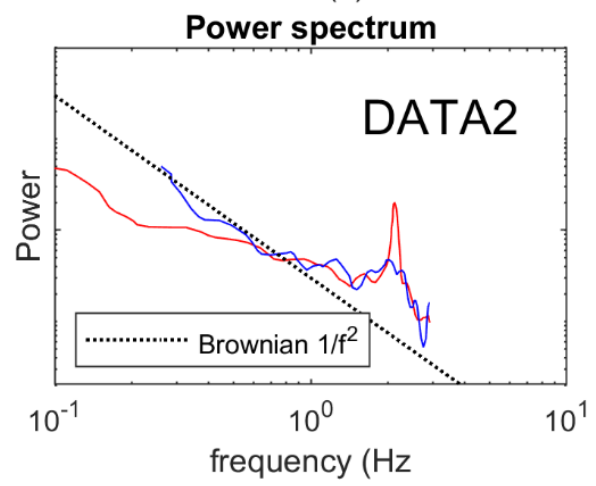

Figure 16. AB: Example accommodation traces in (red) closed-loop response to 1D and (blue) open-loop mode. Dashed

1148 horizontal lines show (red) the 1D demand and (blue) the 1.4D rest focus. A: trace over 5 minutes, to show slow fluctuations in open-loop response; $B$ : 10 s excerpt from $A$, to facilitate comparison with C: Example 10s trace recorded from a human observer, digitised from Fig 3 of Gray, Winn and Gilmartin (Gray et al., 1993a). The red trace is for a 5mm pupil; the blue trace is for viewing through pinholes of $0.5 \mathrm{~mm}$ diameter. A scalebar but no accommodation values are provided in (Gray et al., 1993a), so the vertical position is arbitrary. To facilitate comparison with the model, we have set the mean value to 1D for the closed-loop and 1.4D for the open-loop trace. D: Power spectra of the closed-and open-loop response, obtained by averaging the Fourier power spectra of 50 traces like those in A, generated from simulations with different noise seeds. For comparison, a 1/f2 Brownian noise spectrum is drawn on with a black dashed line. E: Power spectra of closed-and open-loop responses for a human observer, digitised from Fig 5 of (Campbell et al., 1959b). This is labelled DATA2 to make clear that it is not the power spectrum of the trace shown in Figure 16C. No vertical axis scale was provided in (Campbell et al., 1959b), so we have scaled the spectrum so it best agrees with $D$. The red curve was recorded with a $7 \mathrm{~mm}$ pupil and the blue curve with a $1 \mathrm{~mm}$ effective entrance pupil. Code to generate this figure is in Fig_Noise.m; Run_Noise.m must be run first to generate the data. 
1163 Response to step changes

1164 When motivating the introduction of the saturating non-predictive proportional signal, i.e. a

1165 proportional controller responding to the current defocus signal (Figure 11), we discussed why

1166 it produces a larger, more rapid response to small changes in demand. We have already seen

1167 how this effect produces a higher gain for high-frequency low-amplitude oscillations (Figure

1168 13) and thus the ability to track low-amplitude oscillations out to higher temporal frequencies

1169 than is possible for larger amplitudes (Figure 15). Similarly, the non-predictive proportional

1170 signal, clipped at $\pm 0.15 \mathrm{D}$, enables a faster response to small step changes in demand.

1172 Figure 17 demonstrates this by comparing results from the full model (blue) with those from a 1173 model identical except that it lacks the non-predictive proportional signal (orange). To enable 1174 the effects to be seen clearly, noise is also turned off in this simulation. On the left, Figure 1175 17AC, we plot the accommodation and velocity for a $0.5 \mathrm{D}$ increase in demand. The model with 1176 the non-predictive proportional signal responds more quickly. We also see the characteristic 1177 ringing, which is of course what drives the high-frequency peak in the microfluctuations. 1178 However, for the larger 2D step shown on the right (note different $y$-scales), the saturation of 1179 the non-predictive proportional signal at $0.15 \mathrm{D}$ limits its effect, and it makes barely any 1180 difference either to accommodation itself or to velocity. In fact, for large step changes like that 1181 shown in Figure 17BD, there appears to be a fifth signal, a nonlinear pulse triggered by sudden 1182 large changes in demand (Schor \& Bharadwaj, 2006, 2004). The pulse accounts for the 1183 empirical observation that the peak acceleration of the response for step increases in demand 1184 is roughly independent of the step size, instead of scaling with step size as would occur for a 1185 linear system. While implementing the pulse is beyond the scope of this paper, we note that the 1186 non-predictive proportional signal already moves in the right direction by boosting the 1187 acceleration for small steps, and thus helping reduce the difference between acceleration for 1188 large and small steps. This could be another reason for the accommodative control system to 1189 include the postulated non-predictive proportional signal. 

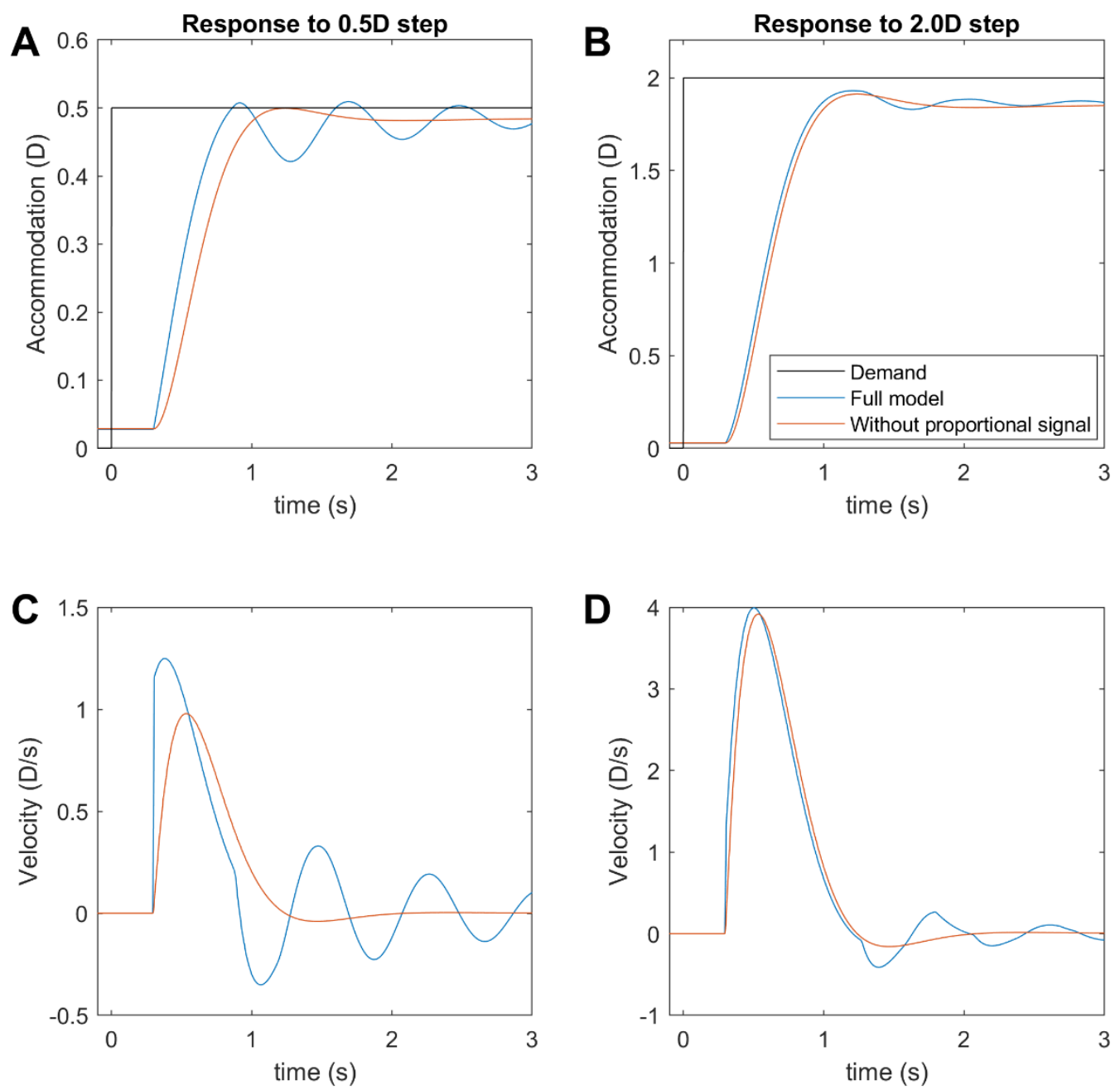

1191 Figure 17. Noise-free accommodation $(A B)$ and velocity $(C D)$ for two different step increases in demand (AC: $0.5 D, B D: 2 D)$.

1192 The blue curve is for the usual model; the orange curve is for a similar model with no non-predictive proportional signal. To 1193 enable the effect to be seen clearly, noise has been turned off for this figure only. Also note that the response to the $2 D$ step

$1194(B D)$ is included only to demonstrate the role of the non-predictive proportional signal. The model presented in this paper 1195 does not accurately capture the dynamics of the response to such large steps, since it does not include the pulse signal (see 1196 text). Code to generate this figure is in Fig_EffectOfPropSignal.m.

1198 Adaptation

1199 Next, we examine how the model adapts to accommodative demand to which it is exposed for 1200 more than a few tens of seconds. This was the motivation for postulating the slow integrator 1201 (Schor, 1979b; Schor et al., 1986). Its presence has not contributed to the results presented so 1202 far, other than to boost the gain for very slow oscillations. Now we see how it accounts for 1203 adaptation. 
1204 Figure 18 shows the time course of accommodation following the application of pinholes at $1205 \mathrm{t}=0$, shifting the system from closed-loop to open-loop demand. After the application of 1206 pinholes, accommodation eventually ends up at the rest focus, but how rapidly it does so 1207 depends on the demand before pinholes were applied. The model observer is initially adapted 1208 to $0 \mathrm{D}$, then switches to viewing 3D for variable amounts of time as shown in the legend. The 1209 results show that after viewing one demand for at least two minutes, the observer adapts to it 1210 such that accommodation remains close to the adapted value for several minutes after pinholes 1211 have been applies (uppermost/red, lowermost/blue traces). Conversely, when the observer was 1212 exposed to different demands immediately before pinholes are applied (middle traces), they 1213 move much more rapidly to the rest focus.

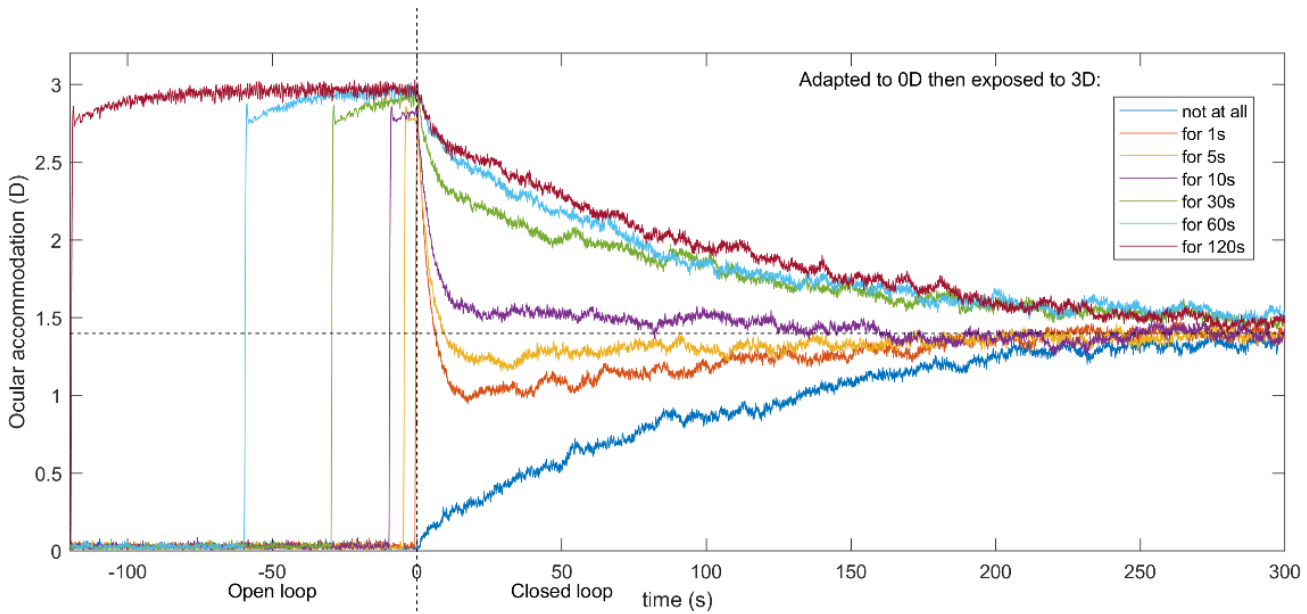

Figure 18. The model shows adaptation to demand, due to the slow integrator. The model observer is initially viewing an

1217 object at $O D$, before then viewing an object at $3 D$ for varying durations as shown in the legend. Pinholes are applied at $t=0 S$,

1218 putting the system in open-loop mode. After long exposures, accommodation adapts to the demand, and moves only slowly

1219 to the rest focus; the adaptation affects the accommodation for many minutes after pinholes have been applied (e.g. dark 1220 blue curve: adapted to $O D$, further than rest focus; red curve: adapted to 3D, closer than rest). Code to generate this figure is in Fig_Adaptation.m; Run_Adaptation.m must be run first to save the data in Results_Adaptation.mat.

1225 Figure 19 shows a comparison with empirical data. Here, the observer was exposed to a demand of $2 \mathrm{D}$ for either $5 \mathrm{~s}$ (blue) or $60 \mathrm{~s}$ (orange) before moving to open-loop mode at $\mathrm{t}=0$. The traces in Figure 19A are for a human observer (Schor et al., 1986); those in Figure 19B are from the model, with rest focus set to $0.4 \mathrm{D}$ (dashed line) in order to better match this observer. In both 
cases, following the $5 \mathrm{~s}$ exposure to $2 \mathrm{D}$, accommodation falls rapidly once the system enters open-loop mode, but following the 60s exposure, the decay is much slower.

1231

1232

1233

1234

1235

1236

1237

1238

1239

1240

1241

1242

1243 Finally, Figure 20 shows the model's steady-state error. As discussed (Equation 16), this

1244 reflects both the fast and slow integrator. In the model, the additional gain provided by the slow

1245 integrator means that steady-state error eventually becomes extremely small. Figure 21 shows

1246 this process for an example step up to 2D. The error is zero at the resting focus but shows

$1247 \mathrm{lag} / \mathrm{lead}$ on either side of this. The gain (response/demand) therefore becomes high as demand tends to zero. 

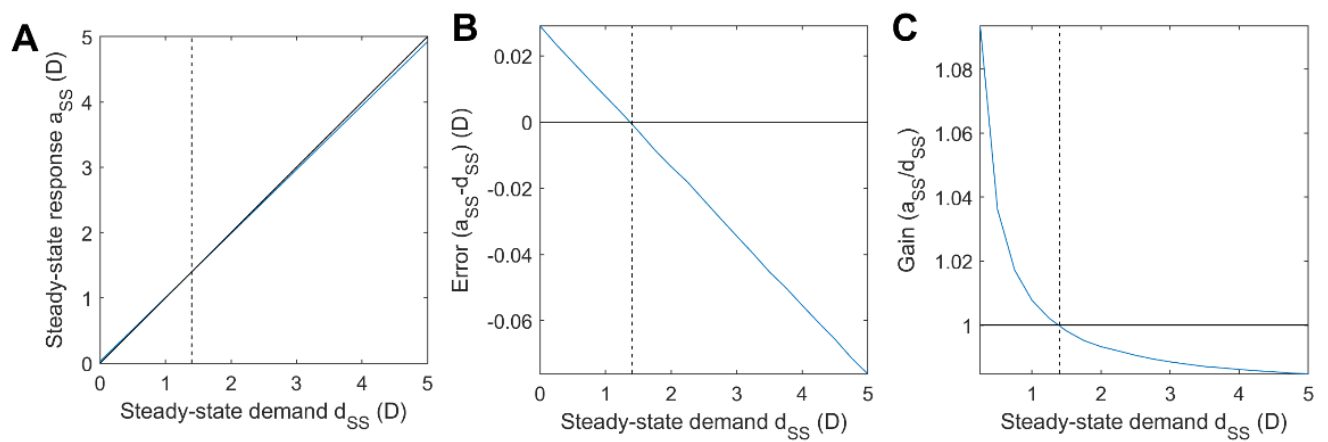

Figure 20. Steady-state response of the model. The model was run for 320 s with a constant demand $d_{s s}$ indicated by the value on the $x$-axis, and accommodation was averaged over the final 60 s to obtain the steady-state response, $a_{s s .}$ (A) Input/output

function, i.e. steady-state accommodation as a function of demand. (B) Steady-state error, i.e. difference between response and demand. For distant stimuli, this is positive (lead); for near, it is negative (lag). (C): Gain, i.e. ratio of response to demand. In each case, blue curves show the response of the model; solid black line indicates response equal to demand, and the dashed vertical line marks the rest focus, where this occurs. Code to generate this figure is in Fig_SteadyState.m; Run_SteadyState

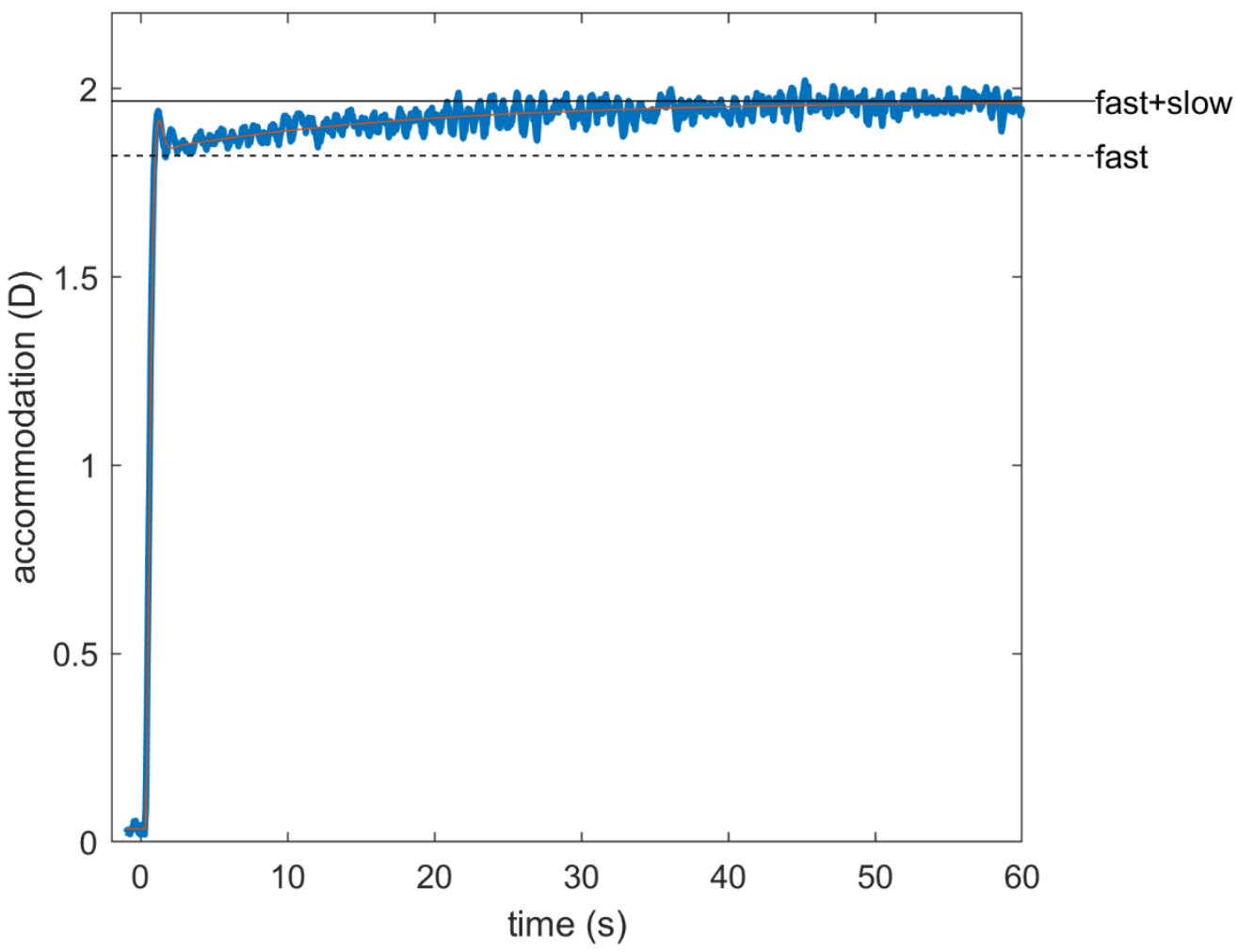

time (s)

Figure 21. Model response to a step change in demand from $O D$ to $2 D$, showing the immediate rise to $89 \%$ of demand due to the fast integrator, and the subsequent slow rise to 98\% demand due to the slow integrator. The blue trace is one example run from the full model; the superimposed orange line shows the response with no noise or non-predictive proportional signal, 
1268 In this paper, we have discussed the neural control of accommodation. We have provided a tutorial overview of the relevant control theory and key empirical observations. We have discussed the evidence for a predictive control system, i.e. one incorporating a forward model to predict the accommodative response in advance of the motor latency (Hung et al., 2002; Khosroyani \& Hung, 2002; Schor \& Bharadwaj, 2004). Our analysis has led us to make the novel proposal that a saturating non-predictive proportional-control component may operate in parallel to the main predictive integrative-control feedback loop. This non-predictive proportional signal causes a high-frequency resonance in the closed-loop response, observed in the response to low-amplitude sinusoidal oscillations in demand. It amplifies noise within the system, explaining the high-frequency peak observed in closed-loop but not open-loop accommodation microfluctuations. It also speeds up the response to small, sudden changes in demand. Yet its saturation means that it does not destabilize the system as a whole, and that it becomes insignificant for large changes in demand.

1282 We have implemented these ideas in a Simulink model, and are publishing this and all code 1283 along with the paper. Although most of the components of the model have been published before, we believe that this model is the first to incorporate realistic sensorimotor latencies, non-zero rest focus, noise and dual control by fast and slow integrators, as well as our novel use of a non-predictive proportional-control signal. Accordingly, it is able to account well for

1287 a wide range of empirical observations: the gain and phase of the response to sinusoidal 1288 oscillations in demand, including the puzzling high-frequency low-frequency resonance; the 1289 power spectrum of microfluctuations in closed-loop and open-loop modes, and the adaptation of accommodation to a steady stimulus.

1292 In our model, accommodation is controlled by four separate signals (Figure 11), which offer 1293 different benefits. The constant bias signal sets the rest focus, to which the system returns in 1294 the absence of other stimulation (Figure 18). This may represent a typical demand, making it easier for the system to respond when stimulation restarts. The slow integrator means that the 
system tends to adapt to steady demand, perhaps reducing disruption if vision is briefly interrupted during sustained attention to one distance.

1299 The fast integrator is the main workhorse of the feedback loop, enabling accommodation to

1300 respond rapidly yet smoothly to changes in demand (Figure 12, Figure 21). It is embedded 1301 within a predictive control system, incorporating a forward model to predict the effect of signals previously sent to the plant. This predictive control enables a smooth response and avoids ringing and instability. In principle, it can entirely remove delay due to the sensorimotor latency in a situation where demand can be predicted perfectly, as in a regular oscillation. However, it becomes problematic where demand changes suddenly and unpredictably.

The fourth signal, proportional to the current defocus, can facilitate rapid responses in such situations (Figure 17). Clipping it at a low absolute value ensures that it does not cause disastrous oscillations in response. Yet its limited instability in closed-loop generates a periodic structure to microfluctuations (Figure 16), which may assist the system in hunting for the point of clearest vision. The benefit of having this periodic structure in closed-loop but not open-

1312 loop mode may be because open-loop viewing occurs naturally for bright viewing conditions, 1313 where the pupil stops down and depth of focus is large. Microfluctuations due to noise 1314 resonances would then be of no assistance in improving vision, and might even cause ocular 1315 fatigue. Presumably for this reason, the system has evolved to select control parameters that avoid resonances in open-loop viewing.

1318 "Prediction" in the accommodation literature has often concentrated on predicting changes in 1319 demand (Krishnan et al., 1973; Stark, 1968). We believe it is helpful to draw a clear distinction 1320 between predicting one's own accommodation, which is in principle possible perfectly with an 1321 efferent copy and a forward model, and predicting demand, which is external and thus not 1322 always possible, for example when a fixated object moves suddenly. Predicting 1323 accommodation but simply using the current demand suffices to achieve closed-loop stability.

1324 The additional benefit of predicting future demand accurately is to avoid delay and thus avoid errors for rapidly changing stimuli. However, the low-pass characteristics of the plant and

1326 leaky-integral controller mean that the benefits of demand prediction are limited unless one 1327 also posits a different form of control. 
1329 The model as currently implemented has many omissions. First, we have not attempted a

1330 realistic implementation of the demand-prediction model. There is some evidence that the brain

1331 can predict changing accommodative demand some time into the future, but we have here

1332 assumed it simply assumes demand will stay constant (Khosroyani \& Hung, 2002). Second,

1333 the model developed here cannot account for the dynamics of step changes, since it does not

1334 include the "pulse" signal triggered by large step changes in accommodation (Schor \&

1335 Bharadwaj, 2006, 2004), which temporarily overrides the error-driven signal. This of course

1336 means that the model presented here cannot accurately model the dynamics of the 1337 accommodative response to such changes. However, the model should remain valid for

1338 situations which do not trigger a pulse (all the situations modelled in the Results, except Figure

1339 17BD, included for illustrative purposes). Third, this paper is purely about motor control, and

1340 has nothing to say about how a signed estimate of defocus is obtained from the retinal image.

1341 Fourth, we do not allow for physical limits on accommodation, a non-zero far point or

1342 refractive error, nor do we consider how the system parameters may change with age

1343 (Bharadwaj \& Schor, 2005; Schor \& Bharadwaj, 2005). Fifth, we do not consider control

1344 signals driven by inputs other than retinal defocus and bias (Heath, 1956b; Maddox, 1893).

1345 Notably, we do not include the crosslinks from and to the vergence system (Bharadwaj, 2005;

1346 Schor \& Kotulak, 1986). We also do not consider other noise sources, such as heartbeat. We

1347 hope to address these deficiencies in future work.

1351 Financial disclosure and competing interests

1352 This work was part-funded by Magic Leap Inc. by a consultancy contract to Newcastle 1353 University for the work of JCAR and CKR. Authors BV and BW are employees of Magic Leap 1354 who initiated the study, reviewed the models and assisted with the preparation of the manuscript. 


\begin{tabular}{|c|c|}
\hline Symbol & Meaning \\
\hline$\omega$ & Angular temporal frequency, $\omega=2 \pi \mathrm{f}$ \\
\hline$\phi(f)$ & phase-delay of accommodation at frequency $f$ \\
\hline$\tau_{\text {fast }}$ & Time-constant of the fast leaky-integrator controller, see Equation 12 \\
\hline$\tau_{\text {plant }}$ & $\begin{array}{l}\text { Time-constant of the ocular plant, when this is modelled as a leaky } \\
\text { integrator, see Equation } 11\end{array}$ \\
\hline$\tau_{\text {slow }}$ & $\begin{array}{l}\text { Time-constant of the slow leaky-integrator controller, see Equation } \\
14\end{array}$ \\
\hline$\zeta$ & Damping coefficient, see Equation 22 \\
\hline$\hat{a}\left(t+T_{m o t}\right)$ & $\begin{array}{l}\text { Predicted accommodation at a time } \mathrm{T}_{\text {mot }} \text { after the current time } \mathrm{t} \text {. In } \\
\text { this paper, generally assumed equal to } \mathrm{a}\left(\mathrm{t}+\mathrm{T}_{\mathrm{mot}}\right) \text {, i.e. prediction is } \\
\text { perfect. }\end{array}$ \\
\hline$\hat{d}\left(t+T_{m o t}\right)$ & $\begin{array}{l}\text { Predicted demand at a time } \mathrm{T}_{\text {mot }} \text { after the current time } \mathrm{t} \text {. In the no- } \\
\text { change prediction model, this is assumed to be the same as the last } \\
\text { available demand, from time } \mathrm{T}_{\text {sens }} \text { before the current time, i.e. } \mathrm{d}(\mathrm{t}- \\
\left.\mathrm{T}_{\text {sens }}\right)\end{array}$ \\
\hline$A(s)$ & Laplace transform of accommodation relative to rest focus \\
\hline$a_{\mathrm{RF}}$ & $\begin{array}{l}\text { Rest focus, i.e. accommodation adopted in the absence of any visual } \\
\text { stimulus }\end{array}$ \\
\hline$a_{\mathrm{SS}}$ & Steady-state accommodation in response to $\mathrm{d}_{\mathrm{SS}}$, see Equation 6 \\
\hline$C(s)$ & Transfer function of controller \\
\hline$D(s)$ & Laplace transform of accommodative demand relative to rest focus \\
\hline$d_{\mathrm{SS}}$ & Steady-state demand, see Equation 6 \\
\hline$E(s)$ & Laplace transform of defocus error, $\mathrm{E}(\mathrm{s})=\mathrm{D}(\mathrm{s})-\mathrm{A}(\mathrm{s})$ \\
\hline$f$ & Temporal frequency \\
\hline$g(f)$ & gain of accommodation at frequency $f$ \\
\hline$G_{\text {fast }}$ & $\begin{array}{l}\text { Steady-state gain of the fast leaky-integrator controller, see Equation } \\
12\end{array}$ \\
\hline$G_{\text {open }}$ & steady-state open-loop gain of accommodation \\
\hline
\end{tabular}




\begin{tabular}{|c|c|}
\hline$G_{\text {slow }}$ & $\begin{array}{l}\text { Steady-state gain of the slow leaky-integrator controller, see } \\
\text { Equation } 14\end{array}$ \\
\hline$H_{\text {closed }}(s)$ & $\begin{array}{l}\text { Closed-loop transfer function relating demand to accommodation, } \\
\text { see Equation } 4\end{array}$ \\
\hline$H_{\text {open }}(s)$ & $\begin{array}{l}\text { Open-loop transfer function relating demand to accommodation, see } \\
\text { Equation } 3\end{array}$ \\
\hline $\bar{j}$ & Square root of -1 . \\
\hline$P(s)$ & Transfer function of ocular plant \\
\hline$S$ & $\begin{array}{l}\text { Complex temporal frequency in Laplace domain, } s=j \omega \text {, see } \\
\text { Equation } 1\end{array}$ \\
\hline$t$ & Time \\
\hline$T_{\text {lat }}$ & Total sensorimotor latency, $\mathrm{T}_{\text {lat }}=\mathrm{T}_{\text {sens }}+\mathrm{T}_{\mathrm{mot}}$ \\
\hline$T_{\text {mot }}$ & $\begin{array}{l}\text { Motor latency, i.e. time taken for the neural signal controlling } \\
\text { accommodation to travel from the brain to the ocular plant }\end{array}$ \\
\hline$T_{\text {sens }}$ & $\begin{array}{l}\text { Sensory latency, i.e. time taken for defocus at the retina to reach the } \\
\text { accommodative control system in the brain }\end{array}$ \\
\hline
\end{tabular}




\begin{tabular}{|c|c|c|c|}
\hline Parameter & $\begin{array}{l}\text { Symbol used in the } \\
\text { paper }\end{array}$ & $\begin{array}{l}\text { Name in Simulink } \\
\text { workspace }\end{array}$ & Value \\
\hline Rest focus & $a_{\mathrm{RF}}$ & RestFocus & $1.4 \mathrm{D}$ \\
\hline Sensory latency & $\mathrm{T}_{\text {sens }}$ & SensoryLatency & $0.20 \mathrm{~s}$ \\
\hline Motor latency & $\mathrm{T}_{\mathrm{mot}}$ & MotorLatency & $0.10 \mathrm{~s}$ \\
\hline $\begin{array}{l}\text { Time constant of } \\
\text { plant }\end{array}$ & $\tau_{\text {plant }}$ & PlantTimeConstant & $0.156 \mathrm{~s}$ \\
\hline $\begin{array}{l}\text { Gain of fast } \\
\text { integrator }\end{array}$ & $\mathrm{G}_{\text {fast }}$ & FastGain & 8.0 \\
\hline $\begin{array}{l}\text { Time constant of } \\
\text { fast integrator }\end{array}$ & $\tau_{\text {fast }}$ & FastTimeConstant & $2.5 \mathrm{~s}$ \\
\hline $\begin{array}{l}\text { Gain of slow } \\
\text { integrator }\end{array}$ & $\mathrm{G}_{\text {slow }}$ & SlowGain & 5.0 \\
\hline $\begin{array}{l}\text { Time constant of } \\
\text { slow integrator }\end{array}$ & $\tau_{\text {slow }}$ & SlowTimeConstant & $100 \mathrm{~s}$ \\
\hline Noise power & & NoisePower & $\begin{array}{l}0.001 \text { with sample } \\
\text { time } 0.01 \mathrm{~s}\end{array}$ \\
\hline $\begin{array}{l}\text { Where to clip the } \\
\text { proportional signal }\end{array}$ & & ProportionalClipping & $0.15 \mathrm{D}$ \\
\hline
\end{tabular}


1371 Here, we derive the total transfer function corresponding to the three types of linear models

1372 discussed in the text: (i) the non-predictive model and the predictive models with (ii) perfect

1373 and (iii) no-change prediction of demand. The bias signal due to the rest focus $a_{R F}$ is included

1374 as an inhomogeneous "forcing" term. We handle this by defining $A(s)$ and $D(s)$ to be the

1375 Laplace transforms of $a(t)-a_{\mathrm{RF}}$ and $d(t)-a_{\mathrm{RF}}$, respectively, where $a(t)$ and $d(t)$ are

1376 accommodation and demand as functions of time. In this way, we can effectively ignore $a_{R F}$ when obtaining the transfer functions.

\section{(i) Non-predictive model}

1380 The system diagram for this model is given in Figure 5. Reading around this circuit diagram,

we see immediately that

$$
E(s)=D(s)-A(s),
$$

1383 where $\mathrm{E}(\mathrm{s})$ is the Laplace transform of the defocus error signal, $d(t)-a(t)$. The input to the 1384 Controller block is $E(s) \exp \left(-s T_{\text {sens }}\right)$, i.e. the defocus error signal after the sensory latency.

1385 The output from the Controller block is $C(s) E(s) \exp \left(-s T_{\text {sens }}\right)$, where $\mathrm{C}(\mathrm{s})$ is the transfer 1386 function of the Controller. After accounting for the motor latency, the input to the ocular plant 1387 is $C(s) E(s) \exp \left(-s T_{\text {lat }}\right)$. So, the output of the ocular plant, i.e. accommodation, is

Substituting in for E(s), we obtain the closed-loop transfer function

$$
A(s)=H_{\text {plant }}(s) C(s) E(s) \exp \left(-s T_{\text {lat }}\right)
$$

$$
H_{\text {closed }}^{\text {classic }}(s)=\frac{A(s)}{D(s)}=\frac{P(s) C(s) \exp \left(-s T_{\text {lat }}\right)}{1+P(s) C(s) \exp \left(-s T_{\text {lat }}\right)}
$$

1391 The gain and phase of the accommodative response to sinusoidal stimuli are the amplitude and phase of the complex number given by this closed-loop transfer function evaluated at $\mathrm{s}=\mathrm{j} \omega=2 \pi \mathrm{j} f, \quad H_{\text {closed }}(2 \pi j f)$. The closed-loop gain as a function of demand frequency is

1394 therefore

$$
G_{\text {closed }}^{\text {classic }}(f)=\frac{|P C|}{\sqrt{1+2 \operatorname{Re}\left(P C e^{\left.-2 \pi j f T_{\text {lat }}\right)+|P C|^{2}}\right.}}
$$

where the plant and controller transfer functions are similarly complex functions of frequency: $P=P(2 \pi j f), \mathrm{C}=C(2 \pi j f)$. The denominator contains oscillatory terms which mean that, even if $P C$ is lowpass (i.e. a monotonically decreasing function of frequency), the denominator can be close to zero at particular frequencies and thus produce large resonances, for which the closed- 
1401 loop gain exceeds 1 . These manifest themselves as ringing or instability in the response to step 1402 changes in demand, and as gains $>1$ for sinusoidal oscillations in demand, which are not 1403 observed for large amplitudes.

1405 With proportional control with unit gain $(\mathrm{C}=1)$, a sensorimotor latency of $\mathrm{T}_{\text {lat }}=0.3 \mathrm{~s}$ and the 1406 plant being a leaky integrator with $\tau_{\text {plant }}=0.156 \mathrm{~s}$, Equation 17 has its first resonance at $1.2 \mathrm{~Hz}$ 1407 where the closed-loop gain goes well above 1. This is ultimately responsible for the model's 1408 high-frequency peak in microfluctuations (Figure 16) and the low-amplitude resonance in the 1409 response to sine-waves (Figure 13), although the precise behaviour also depends on the 1410 nonlinear clipping. The precise position of the first resonance depends on the gain of the 1411 proportional control, but only rather subtly. We therefore kept unit gain for simplicity.

1412 We obtain the open-loop transfer function in the same way, but with the input to the Controller 1413 being $\mathrm{D}(\mathrm{s})$ instead of $\mathrm{D}(\mathrm{s})-\mathrm{A}(\mathrm{s})$. This yields

$$
\begin{gathered}
H_{\text {open }}^{\text {classic }}(s)=P(s) C(s) \exp \left(-s T_{\text {lat }}\right) \\
G_{\text {open }}^{\text {classic }}(\omega)=|P C|
\end{gathered}
$$

1417 Thus, whether we use an integral or proportional controller in this non-predictive control 1418 system, the open-loop gain is purely low-pass, with no resonances. This means that adding our 1419 non-predictive proportional signal does not introduce any peaks to the power spectrum of open1420 loop microfluctuations.

\section{Predictive models}

1423 The simplified system diagram for this model is given in Figure 7. As usual, we can ignore the 1424 bias signal if we express accommodation and demand relative to the rest focus. Reading around 1425 the circuit diagram, the demand signal is the input on the left; we represent this as usual in the 1426 Laplace domain by $\mathrm{D}(\mathrm{s})$. After passing through the sensory latency, it becomes $1427 D(s) \exp \left(-s T_{\text {sens }}\right)$, with the exponential being the Laplacian representation of a time delay 1428 (cf discussion of Equation 2). It then passes through the demand predictor, which attempts to 1429 predict the signal $T_{\text {lat }}=T_{\text {sens }}+T_{m o t}$ into the future. If it did this perfectly, the output of the demand 1430 predictor would be $D(s) \exp \left(-s T_{\text {sens }}\right) \exp \left(-s T_{\text {lat }}\right)=D(s) \exp \left(+s T_{\text {mot }}\right)$. To allow for the 1431 fact that demand is unlikely to be predicted perfectly, we will write the output as $1432 \widehat{D}(s) \exp \left(+s T_{m o t}\right) . \widehat{D}(s)$ is the Laplace transform of the estimated future demand, again 
relative to the rest focus. That is, whereas $d(t)$ is the actual demand at time $t, \hat{d}(t)$ is the estimated demand at time $t$, as estimated at time $\left(t-T_{\text {lat }}\right)$.

1435 Looking at the bottom of Figure 7, the output is accommodation, or A(s) in the Laplace domain.

1436 This is output after a motor latency $\mathrm{T}_{\text {mot}}$; thus the output of the "Plant" block in Figure 7 is $1437 A(s) \exp \left(+s T_{\text {mot }}\right)$.

1438 Putting both these together, we see that the input to the Controller in Figure 7 is $[\widehat{D}(s)-A(s)] \exp \left(+s T_{m o t}\right)$. After multiplying this by the Controller and Plant transfer

1440 functions, we find that the output of the plant is $P(s) C(s)[\widehat{D}(s)-A(s)] \exp \left(+s T_{m o t}\right)$. But we 1441 previously saw that the output of the plant is $A(s) \exp \left(+s T_{\text {mot }}\right)$. Equating these, we see that

$$
A(s)=P(s) C(s)[\widehat{D}(s)-A(s)]
$$

1443 and thus that

$$
A(s)=\frac{P(s) C(s) \widehat{D}(s)}{1+P(s) C(s)}
$$

(ii) Perfect demand predictor

1448 In this idealised case, the demand predictor successfully outputs the future accommodative demand, so that

$$
\begin{gathered}
\widehat{D}(s)=D(s) \quad \text { and the } \\
H_{\text {closed }}^{\text {perfect }}(s)=\frac{P(s) C(s)}{1+P(s) C(s)}
\end{gathered}
$$

1451 The closed-loop

gain is therefore

$$
g_{\text {closed }}^{\text {perfect }}(f)=\frac{|P C|}{\sqrt{1+2 \operatorname{Re}(P C)+|P C|^{2}}}
$$

To obtain the open-loop transfer function, we replace $\mathrm{D}(\mathrm{s})$ with $\mathrm{D}(\mathrm{s})+\mathrm{A}(\mathrm{s})$ in Equation 19 (Figure 4), obtaining

and thus

$$
A(s)=\frac{P(s) C(s)[A(s)+D(s)]}{1+P(s) C(s)}
$$

$$
H_{\text {open }}^{\text {perfect }}(s)=P(s) C(s)
$$

If demand prediction is perfect, the open-loop gain of the controller is independent of latency. For our situation where both the plant and controller are leaky integrators, the open-loop gain 
1463 In this opposite extreme, the demand predictor simply assumes that the future defocus after

1464 time $\mathrm{T}_{\text {lat }}$ will still be the same as the defocus it is receiving now (Equation 9):

$$
\hat{d}\left(t+T_{\text {lat }}\right)=d(t)
$$

1466 and thus

$$
\widehat{D}(s)=D(s) \exp \left(-s T_{\text {lat }}\right)
$$

1468 Hence

$$
H_{\text {closed }}^{\text {nochange }}(s)=\frac{P(s) C(s) \exp \left(-s T_{\text {lat }}\right)}{1+P(s) C(s)}
$$

The closed-loop gain at any frequency $f$ is therefore the same as for the perfect predictor, while the phase is reduced by $2 \pi f \Gamma_{\text {lat. }}$ In fact, the closed-loop gain would be the same for any demand predictor which accurately predicts demand any time at all into the future, even if, as here, that time is zero. Inaccurate predictions would of course change the closed-loop gain.

The open-loop gain does depend critically on demand prediction. With no-change prediction, replacing $\mathrm{D}(\mathrm{s})$ with $\mathrm{D}(\mathrm{s})+\mathrm{A}(\mathrm{s})$ in Equation 19 (Figure 4), yields

$$
A(s)=\frac{P(s) C(s)[A(s)+D(s)] \exp \left(-s T_{\text {lat }}\right)}{1+P(s) C(s)}
$$

and thus

$$
H_{\text {open }}^{\text {nochange }}(s)=\frac{P(s) C(s) \exp \left(-s T_{\text {lat }}\right)}{1+P(s) C(s)\left(1-\exp \left(-s T_{\text {lat }}\right)\right)}
$$

1481 The presence of the oscillatory $\exp \left(-s T_{\text {lat }}\right)$ term in the denominator can lead to local peaks in the gain at some frequencies. Thus with inaccurate no-change prediction, the system is prone to open-loop resonances due to the inner feedback loop via the efference copy. However, with our parameter values (Table 2), Equation 20 is a monotonically decreasing function of frequency. This ensures that we do not see local peaks in the power spectrum of open-loop microfluctuations (Figure 16). 


$$
H_{\text {closed }}^{\text {perfect }}(s)=\frac{G_{\text {fast }}}{\left(1+s \tau_{\text {plant }}\right)\left(1+s \tau_{\text {fast }}\right)+G_{\text {fast }}}
$$

1494 with $s=2 \pi j f$. This is the transfer function of a second-order damped oscillator. We can rewrite 1495 it in the standard form

$$
H_{\text {closed }}^{\text {perfect }}(s) \approx \frac{K \omega_{0}^{2}}{s^{2}+2 \zeta \omega_{0} s+\omega_{0}^{2}}
$$

1497 where $\mathrm{K}$ is the closed-loop gain:

$$
K=\frac{G_{\text {fast }}}{\left(1+G_{\text {fast }}\right)}
$$

$\omega_{0}$ the natural angular frequency:

$$
\omega_{0}^{2}=\frac{\left(1+G_{\text {fast }}\right)}{\tau_{\text {plant }} \tau_{\text {fast }}}
$$

and $\zeta$ the damping coefficient:

$$
\zeta=\frac{1}{2 \sqrt{1+G_{\text {fast }}}} \frac{\left(\tau_{\text {plant }}+\tau_{\text {fast }}\right)}{\sqrt{\tau_{\text {plant }} \tau_{\text {fast }}}}
$$

1504 For perfect demand prediction, the phase at angular frequency $\omega$ is:

$$
\phi^{\text {perfect }}(w)=-\arctan \left(\frac{2 \zeta \omega \omega_{0}}{\omega_{0}^{2}-\omega^{2}}\right)
$$

1506 while for no-change prediction,

$$
\phi^{\text {nochange }}(\omega)=-\arctan \left(\frac{2 \zeta \omega \omega_{0}}{\omega_{0}^{2}-\omega^{2}}\right)-\omega T_{\text {lat }}
$$

1509 If $\zeta<1 / \sqrt{ } 2$, then the maximum gain occurs at the resonant angular frequency:

$$
\omega_{\text {res }}=\omega_{0} \sqrt{1-2 \zeta^{2}}=\sqrt{\frac{G_{\text {fast }}}{\tau_{\text {plant }} \tau_{\text {fast }}}-\frac{1}{2 \tau_{\text {fast }}^{2}}-\frac{1}{2 \tau_{\text {plant }}^{2}}}
$$

1511 If $\zeta>1 / \sqrt{2}$, then the gain is maximum for $\mathrm{f}=0$ and decreases monotonically with frequency. If $1512 \zeta=1$, the system is said to be critically damped.

1513 As discussed in the text, to match the empirical gain of accommodation, $\zeta$ must exceed $1 / \sqrt{ } 2$,

1514 the minimum value for which gain decreases monotonically with frequency. Solving Equation

151522 , we find that 
$\tau_{\text {fast }}=\tau_{\text {plant }}\left(G_{\text {fast }}+\sqrt{G_{\text {fast }}^{2}-1}\right) \approx 2 G_{\text {fast }} \tau_{\text {plant }}$ yields $\zeta=1 / \sqrt{2}$, while

$\tau_{\text {fast }}=\tau_{\text {plant }}\left(2 G_{\text {fast }}+1+\sqrt{\left[2 G_{\text {fast }}+1\right]^{2}-1}\right) \approx 4 G_{\text {fast }} \tau_{\text {plant }}$ yields $\zeta=1$, i.e. critical

1518 damping

where the approximations hold since the gain $\mathrm{G}_{\text {fast }}$ has to be $>>1$, say at least 5 , to avoid excessive lag. (Mathematically, there are two solutions, but the other one gives a very short time-constant for the controller, which in turn causes other problems such as open-loop resonances in the noise.)

The minimal-settling time solution

1526 In the model presented here, we chose the "minimum settling time" solution which yields $\zeta=$ $1527 \quad 1 / \sqrt{ } 2$ :

$$
\tau_{\text {fast }}=2 G_{\text {fast }} \tau_{\text {plant }}
$$

1529 since this gave the best match to both gain and phase data. With this choice, since $\mathrm{G}_{\text {fast }}>>1$, the natural frequency is approximately

$$
\omega_{0}=\frac{1}{\tau_{\text {plant }} \sqrt{2}}
$$

which with our value $\tau_{\text {plant }}=0.156 \mathrm{~s}$ corresponds to $0.72 \mathrm{~Hz}$.

1533 For $\zeta=1 / \sqrt{ } 2$, the phase function is very close to linear out to $\omega=\omega_{0}$. In this region, for perfect demand prediction

$$
\phi^{\text {perfect }} \approx-2 \tau_{\text {plant }} \omega
$$

corresponding to an effective delay of $T_{\text {delay }}=2 \tau_{\text {plant. }}$ Presumably coincidentally, this delay is very similar to the sensorimotor latency, although as we can see it arises from a completely different source. However, for frequencies beyond $\sim 1 \mathrm{~Hz}$, the phase asymptotes to $180^{\circ}$ (Figure 8).

For no-change prediction, the phase is approximately

$$
\phi(\omega) \approx-\omega\left(2 \tau_{\text {plant }}+T_{\text {lat }}\right)
$$
at low frequencies, corresponding to an effective delay of $2 \tau_{\text {plant }}+T_{\text {lat }}$. 


\begin{tabular}{|c|c|c|c|}
\hline $\begin{array}{l}\text { Transfer } \\
\text { function } \\
A(s) \\
=H(s) D(s)\end{array}$ & $\begin{array}{l}\text { Non-predictive model }- \text { no } \\
\text { prediction }\end{array}$ & $\begin{array}{l}\text { Predictive model }- \\
\text { perfect prediction of } \\
\text { future demand }\end{array}$ & $\begin{array}{l}\text { Predictive model - } \\
\text { "no change" } \\
\text { prediction of future } \\
\text { demand }\end{array}$ \\
\hline $\begin{array}{l}\text { Open-loop } \\
\text { transfer } \\
\text { function }\end{array}$ & $P C e^{-s T_{l a t}}$ & $P C$ & $\frac{P C e^{-s T_{\text {lat }}}}{1+P C\left(1-e^{-s T_{\text {lat }}}\right)}$ \\
\hline $\begin{array}{l}\text { Closed- } \\
\text { loop } \\
\text { transfer } \\
\text { function }\end{array}$ & $\frac{P C e^{-s T_{\text {lat }}}}{1+P C e^{-s T_{\text {lat }}}}$ & $\frac{P C}{1+P C}$ & $\frac{P C e^{-s T_{\text {lat }}}}{1+P C}$ \\
\hline $\begin{array}{l}\text { Closed- } \\
\text { loop gain }\end{array}$ & $\frac{|P C|}{\sqrt{1+2 \operatorname{Re}\left(P C e^{\left.-i \omega T_{\text {lat }}\right)+\mid P C}\right.}}$ & $\frac{|P C|}{\sqrt{1+2 \operatorname{Re}(P C)+\mid P C}}$ & $\frac{|P C|}{\sqrt{1+2 \operatorname{Re}(P C)+\mid P C}}$ \\
\hline
\end{tabular}

function relates accommodation to the demand via $A(s)=H(s) D(s)$, where $A(s)$ is the Laplace transform of accommodation relative to rest focus, $a(t)-a_{R F}$, and $D(s)$ is the Laplace transform of demand relative to rest focus, $d(t)-a_{R F} . P(s)$ the transfer function of the ocular plant, and $C(s)$ is the transfer function of the neural control (block marked Controller in Figure 5, Figure

\section{References}

Águila-Carrasco, A. J. D., \& Marín-Franch, I. (2021). Predictability of sinusoidally moving stimuli does not improve the accuracy of the accommodative response. Scientific Reports, 11(1), 15195. https://doi.org/10.1038/s41598-021-94642-2

Bahill, A. T., Clark, M. R., \& Stark, L. (1975). Computer simulation of overshoot in saccadic eye movements. 
1560 Beers, A. P. A., \& Van Der Heijde, G. L. (1994). In vivo determination of the biomechanical properties of the component elements of the accommodation mechanism. Vision Research, 34(21), 2897-2905. https://doi.org/10.1016/0042-6989(94)90058-2

Beers, A. P., \& van der Heijde, G. L. (1996). Age-related changes in the accommodation mechanism. Optometry and Vision Science : Official Publication of the American Academy of Optometry, 73(4), 235-242. https://doi.org/10.1097/00006324-199604000-00004

Bharadwaj, S. R. (2005). Neural Control Strategies of the Human Focusing Mechanism.

Bharadwaj, S. R., \& Schor, C. M. (2005). Acceleration characteristics of human ocular accommodation. Vision Research, 45(1), 17-28. https://doi.org/10.1016/j.visres.2004.07.040

Bharadwaj, S. R., \& Schor, C. M. (2006). Dynamic control of ocular disaccommodation: First and second-order dynamics. Vision Res, 46(6-7). https://doi.org/10.1016/j.visres.2005.06.005

Burge, J., \& Geisler, W. S. (2011). Optimal defocus estimation in individual natural images. Proc Natl Acad Sci U S A, 108(40), 16849-16854. https://doi.org/1108491108 [pii] 10.1073/pnas.1108491108

Campbell, F. W., Robson, J. G., \& Westheimer, G. (1959a). Fluctuations of accommodation under steady viewing conditions. The Journal of Physiology, 145(3), 579-594.

Campbell, F. W., Robson, J. G., \& Westheimer, G. (1959b). Fluctuations of accommodation under steady viewing conditions. The Journal of Physiology, 145(3), 579-594.

1580 Campbell, F. W., \& Westheimer, G. (1960). Dynamics of accommodation responses of the human eye. The Journal of Physiology, 151(2), 285-295. https://doi.org/10.1113/jphysiol.1960.sp006438

1582 Charman, W. N., \& Heron, G. (1988). Fluctuations in accommodation: A review. Ophthalmic \& Physiological Optics: The Journal of the British College of Ophthalmic Opticians (Optometrists), 8(2), 153-164. https://doi.org/10.1111/j.1475-1313.1988.tb01031.x 
1585 Charman, W. N., \& Heron, G. (2000). On the linearity of accommodation dynamics. Vision Research, 40(15), 2057-2066. https://doi.org/10.1016/S0042-6989(00)00066-3

1587 Charman, W. N., \& Heron, G. (2015). Microfluctuations in accommodation: An update on their characteristics and possible role. Ophthalmic and Physiological Optics, 35(5), 476-499. https://doi.org/10.1111/opo.12234

Cholewiak, S. A., Love, G. D., \& Banks, M. S. (2018). Creating correct blur and its effect on accommodation. Journal of Vision, 18(9). https://doi.org/10.1167/18.9.1

Collins, M., Davis, B., \& Wood, J. (1995). Microfluctuations of steady-state accommodation and the cardiopulmonary system. Vision Research, 35(17), 2491-2502.

1594 Denieul, P. (1982). Effects of stimulus vergence on mean accommodation response, microfluctuations of accommodation and optical quality of the human eye. Vision Research, 22(5), 561-569. https://doi.org/10.1016/0042-6989(82)90114-6

Ejiri, M., Thompson, H. E., \& O'Neill, W. D. (1969). Dynamic visco-elastic properties of the lens. Vision Research, 9(2), 233-244. https://doi.org/10.1016/0042-6989(69)90003-0

Fincham, E. F. (1951). The Accommodation Reflex and its Stimulus. The British Journal of Ophthalmology, 35(7), 381-393.

Gall, J. (1977). Systemantics: How Systems Work \& Especially How They Fail. Times Books.

Gambra, E., Sawides, L., Dorronsoro, C., \& Marcos, S. (2009). Accommodative lag and fluctuations when optical aberrations are manipulated. Journal of Vision, 9(6), 4.1-15. https://doi.org/10.1167/9.6.4

Gamlin, P. D., Zhang, Y., Clendaniel, R. A., \& Mays, L. E. (1994). Behavior of identified Edinger-Westphal

Gray, L. S., Winn, B., \& Gilmartin, B. (1993a). Effect of target luminance on microfluctuations of accommodation. Ophthalmic \& Physiological Optics: The Journal of the British College of 
Ophthalmic Opticians (Optometrists), 13(3), 258-265. https://doi.org/10.1111/j.14751313.1993.tb00468.x

1612 Gray, L. S., Winn, B., \& Gilmartin, B. (1993b). Accommodative microfluctuations and pupil diameter. Vision Research, 33(15), 2083-2090. https://doi.org/10.1016/0042-6989(93)90007-j

1614 Heath, G. G. (1956a). The influence of visual acuity on accommodative responses of the eye. American Journal of Optometry and Archives of American Academy of Optometry, 33(10), 513-524. https://doi.org/10.1097/00006324-195610000-00001

Heath, G. G. (1956b). Components of accommodation. American Journal of Optometry and Archives of American Academy of Optometry, 33(11), 569-579. https://doi.org/10.1097/00006324-

Heron, G., Charman, W. N., \& Gray, L. S. (1999). Accommodation Responses and Ageing. Investigative Ophthalmology \& Visual Science, 40(12), 2872-2883.

Hung, G. K., Ciuffreda, K. J., Khosroyani, M., \& Jiang, B.-C. (2002). Models of Accommodation (pp. 287339). https://doi.org/10.1007/978-1-4757-5865-8_8

Khosroyani, M., \& Hung, G. K. (2002). A Dual-Mode Dynamic Model of the Human Accommodation System. Bulletin of Mathematical Biology, 64(2), 285-299. https://doi.org/10.1006/bulm.2001.0274

Kotulak, J. C., \& Schor, C. M. (1986a). The accommodative response to subthreshold blur and to perceptual fading during the Troxler phenomenon. Perception, 15(1), 7-15. https://doi.org/10.1068/p150007

Kotulak, J. C., \& Schor, C. M. (1986b). Temporal variations in accommodation during steady-state conditions. Journal of the Optical Society of America. A, Optics and Image Science, 3(2), 223-

1633 Kotulak, J. C., \& Schor, C. M. (1986c). A computational model of the error detector of human visual 
Krishnan, V. V., Phillips, S., \& Stark, L. (1973). Frequency analysis of accommodation, accommodative vergence and disparity vergence. Vision Research, 13(8), 1545-1554.

Krishnan, V. V., \& Stark, L. (1975). Integral control in accommodation. Computer Programs in Biomedicine, 4(4), 237-245. https://doi.org/10.1016/0010-468X(75)90037-9

Kruger, P. B., Mathews, S., Aggarwala, K. R., \& Sanchez, N. (1993). Chromatic aberration and ocular focus: Fincham revisited. Vision Research, 33(10), 1397-1411. https://doi.org/10.1016/0042-

Kruger, P. B., \& Pola, J. (1986). Stimuli for accommodation: Blur, chromatic aberration and size. Vision Research, 26(6), 957-971. https://doi.org/10.1016/0042-6989(86)90153-7

Labhishetty, V., \& Bobier, W. R. (2017). Are high lags of accommodation in myopic children due to motor deficits? Vision Research, 130, 9-21. https://doi.org/10.1016/j.visres.2016.11.001

Leibowitz, H. W., \& Owens, D. A. (1978). New evidence for the intermediate position of relaxed accommodation. Documenta Ophthalmologica. Advances in Ophthalmology, 46(1), 133-147. https://doi.org/10.1007/BF00174103

Maddox, E. E. (1893). The Clinical Use of Prisms; and the Decentering of Lenses, 2nd edition. John Wright \& Sons, Bristol, England. (2nd ed.). John Wright \& Sons.

Miall, R. C., Weir, D. J., Wolpert, D. M., \& Stein, J. (1993). Is the cerebellum a Smith predictor? Journal of Motor Behaviour, 25(3), 203-216.

Ohtsuka, K., \& Sawa, M. (1997). Frequency characteristics of accommodation in a patient with

Otero, C., Aldaba, M., Díaz-Doutón, F., Vera-Diaz, F. A., \& Pujol, J. (2019). Stimulus Unpredictability in agenesis of the posterior vermis and normal subjects. British Journal of Ophthalmology, 81(6), Time, Magnitude, and Direction on Accommodation. Optometry and Vision Science: Official Publication of the American Academy of Optometry, 96(6), 424-433. https://doi.org/10.1097/OPX.0000000000001384 
1661 Phillips, S., Shirachi, D., \& Stark, L. (1972). ANALYSIS OF ACCOMMODATIVE RESPONSE TIMES USING Academy of Optometry, 49(5), 389-400.

Popa, L. S., \& Ebner, T. J. (2019). Cerebellum, Predictions and Errors. Frontiers in Cellular Neuroscience, 12. https://doi.org/10.3389/fncel.2018.00524

Rosenfield, M., Ciuffreda, K. J., Hung, G. K., \& Gilmartin, B. (1993a). Tonic accommodation: A review. Ophthalmic Opticians (Optometrists), 13(3), 266-284.

1669 Rosenfield, M., Ciuffreda, K. J., Hung, G. K., \& Gilmartin, B. (1993b). Tonic accommodation: A review. Ophthalmic Opticians (Optometrists), 13(3), 266-284. https://doi.org/10.1111/j.1475-

Schor, C. M. (1979a). The influence of rapid prism adaptation upon fixation disparity. Vision Research, 19(7), 757-765. https://doi.org/10.1016/0042-6989(79)90151-2

Schor, C. M. (1979b). The relationship between fusional vergence eye movements and fixation disparity. Vision Research, 19(12), 1359-1367. https://doi.org/10.1016/0042-6989(79)90208-

Schor, C. M., \& Bharadwaj, S. R. (2005). A pulse-step model of accommodation dynamics in the aging eye. Vision Research, 45(10), 1237-1254. https://doi.org/10.1016/j.visres.2004.11.011

Schor, C. M., \& Bharadwaj, S. R. (2006). Pulse-step models of control strategies for dynamic ocular accommodation and disaccommodation. Vision Research, 46(1), 242-258.

1683 Schor, C. M., \& Bharadwaj, S. R. (2004). A pulse-step model of accommodation dynamics. The 26th Annual International Conference of the IEEE Engineering in Medicine and Biology Society, 3, 766-769. https://doi.org/10.1109/IEMBS.2004.1403271 
Schor, C. M., \& Kotulak, J. C. (1986). Dynamic interactions between accommodation and convergence are velocity sensitive. Vision Research, 26(6), 927-942. https://doi.org/10.1016/00426989(86)90151-3

Schor, C. M., Kotulak, J. C., \& Tsuetaki, T. (1986). Adaptation of tonic accommodation reduces accommodative lag and is masked in darkness. Investigative Ophthalmology \& Visual Science, $27(5), 820-827$.

Schor, C. M., Lott, L. A., Pope, D., \& Graham, A. D. (1999). Saccades reduce latency and increase velocity of ocular accommodation. Vision Research, 39(22), 3769-3795. https://doi.org/10.1016/s0042-6989(99)00094-2

Seidemann, A., \& Schaeffel, F. (2002). Effects of longitudinal chromatic aberration on accommodation and emmetropization. Vision Research, 42(21), 2409-2417. https://doi.org/10.1016/S0042-

Smith, O. (1957). Closer control of loops with dead time. Chemical Engineering Progress, 53(5), 217219.

Stark, L. (1968). Accommodative Tracking: A Trial-and-Error Function. In L. Stark (Ed.), Neurological Control Systems: Studies in Bioengineering (pp. 220-230). Springer US. https://doi.org/10.1007/978-1-4684-0706-8_10

Stark, L., Takahashi, Y., \& Zames, G. (1965). Nonlinear Servoanalysis of Human Lens Accommodation. IEEE Transactions on Systems Science and Cybernetics, 1(1), 75-83. https://doi.org/10.1109/TSSC.1965.300064

Sun, F., Brandt, S., Nguyen, A., Wong, M., \& Stark, L. (1989). Frequency analysis of accommodation: Single sinusoids. Ophthalmic \& Physiological Optics: The Journal of the British College of

1709 Sun, F. C., \& Stark, L. (1990). Switching control of accommodation: Experimental and simulation responses to ramp inputs. IEEE Transactions on Bio-Medical Engineering, 37(1), 73-79. https://doi.org/10.1109/10.43618 
1712 Toates, F. M. (1972). Accommodation function of the human eye. Physiological Reviews, 52(4).

1713 Udlam, W. M., Wittenberg, S., Giglio, E. J., \& Rosenberg, R. (1968). Accommodative responses to small changes in dioptric stimulus. American Journal of Optometry and Archives of American Academy of Optometry, 45(8), 483-506. https://doi.org/10.1097/00006324-19680800000001

Wildt, G. J. van der, Bouman, M. A., \& Kraats, J. van de. (1974). The Effect of Anticipation on the Transfer Function of the Human Lens System. Optica Acta: International Journal of Optics, 21(11), 843-860. https://doi.org/10.1080/713818858

Wilson, B. J., Decker, K. E., \& Roorda, A. (2002). Monochromatic aberrations provide an odd-error cue to focus direction. Journal of the Optical Society of America A-Optics Image Science and Vision,

Wilson, D. (1973). A centre for accommodative vergence motor control. Vision Research, 13(12), 2491-2503. https://doi.org/10.1016/0042-6989(73)90246-0

Winn, B., Pugh, J. R., Gilmartin, B., \& Owens, H. (1990). Arterial pulse modulates steady-state ocular accommodation.

Current Eye

Research,

9(10),

971-975. https://doi.org/10.3109/02713689009069933

Yao, P., Lin, H., Huang, J., Chu, R., \& Jiang, B. (2010). Objective depth-of-focus is different from subjective depth-of-focus and correlated with accommodative microfluctuations. Vision Research, 50(13), 1266-1273. https://doi.org/10.1016/j.visres.2010.04.011 